\title{
New Photolithography Stepping Machine
}

\author{
Layton Hale: \\ * Jeff Klingmann \\ David Markle
}

March 8, 1995 
This document was prepared as an account of work sponsored by an agency of the United States Government. Neither the United States Government nor the University of California nor any of their employees, makes any warranty, express or implied, or assumes any legal liability or responsibility for the accuracy, completeness, or usefulness of any information, apparatus, product, or process disclosed, or represents that its use would not infringe privately owned rights. Reference herein to any specific commercial products, process, or service by trade name, trademark, manufacturer, or otherwise, does not necessarily constitute or imply its endorsement, recommendation, or favoring by the United States Government or the University of California. The views and opinions of authors expressed herein do not necessarily state or reflect those of the United States Government or the University of California, and shall not be used for advertising or product endorsement purposes.

This report has been reproduced directly from the best available copy.

A vailable to DOE and DOE contractors from the Office of Scientific and Technical Information P.O. Box 62, Oak Ridge, TN 37831

Prices a vailable from (615) 576.8401, FTS 626-8401

Available to the public from the National Technical Information Service

U.S. Department of Commerce 5285 Port Royal Rd. Springfield, VA 22161 


\section{DISCLAIMER}

Portions of this document may be illegible in electronic image products. Images are produced from the best available original document. 


\title{
New Photolithography Stepping Machine
}

\section{Layton Hale}

Lawrence Livermore National Laboratory

Jeff Klingmann

Lawrence Livermore National Laboratory

David Markle ${ }^{1}$

Ultratech Stepper Inc.

\begin{abstract}
A joint development project to design a new photolithography stepping machine capable of 150 nanometer overlay accuracy was completed by Ultratech Stepper and the Lawrence Livermore National Laboratory. ${ }^{2}$ The principal result of the project is a next-generation product that will strengthen the U.S. position in step-and-repeat photolithography. The significant challenges addressed and solved in the project are the subject of this report. Design methods and new devices that have broader application to precision machine design are presented in greater detail while project specific information serves primarily as background and motivation.
\end{abstract}

\section{Introduction}

The semiconductor industry, due to performance driven competition, continues to achieve unprecedented technological advancements. For example, the memory density of DRAM chips increases four fold every three years or so. Advances in integrated circuit technology drive the producers of processing equipment to achieve commensurate advances, or conversely, advances in manufacturing technology allow advances in integrated circuits. A vital U.S. technology addressed by this project is step-and-repeat photolithography. It demands motion control to tens of nanometers, nearly perfect optical systems and competitive throughput of product.

Ultratech Stepper, a company of only 280 employees, is the only U.S. producer of photolithography steppers to compete with the giant Japanese companies, Canon and Nikon. Through a partnership between Ultratech Stepper and the Lawrence Livermore National Laboratory (LLNL), a new photolithography stepper capable of 150 nanometer overlay accuracy was developed, which will strengthen the U.S. position in step-andrepeat photolithography. The ambitious schedule set forth in the business plan required that existing designs, components and processes having sufficient accuracy be used to the greatest extent possible. Preliminary analysis indicated that the step-and-repeat stage of the

\footnotetext{
${ }^{1}$ A number of people under Dave's direction deserve credit for bring this machine into existence. Paul Rasmussen and Ed Louie were key people in the design process of which this report is about.

${ }^{2}$ This work was performed under the auspices of the U.S. Department of Energy by the Lawrence Livermore National Laboratory under contract No. W-7405-Eng-48. See the CRADA document number TSB-842-94 for details on the partnership agreement.
} 
present Ultratech product would meet the next-generation requirements. Thus, efforts were concentrated on the optical projection system (or lens), which images light that passes through the reticle (or mask) onto the semiconductor.

The optical configuration of the new lens is identical to previous Ultratech designs, but the optics are larger to achieve better image resolution. Greater resolution is important to allow smaller features to be printed onto the semiconductor; however, resolution by itself is insufficient for good overlay accuracy, which requires reduction of multiple error sources tending to misalign features between two separately printed layers. An error source may affect overlay accuracy quite differently depending on where it acts in the system and the time scale on which it acts. For example, thermal or manufacturing error sources that cause quasi-static distortion of the primary mirror by several nanometers is significant because each exposure becomes distorted. Rigid body motion of the mirror to this level is not of concern if it occurs over several minutes because the alignment process corrects for the shift of the image. Obviously it is important during the design process to recognize how error sources affect accuracy (or quality) so that resources are directed wisely.

With the optical design set and the long-lead optical components on order, Ultratech and LLNL engineers worked together to iterate on the best design concept for supporting the optics relative to the step-and-repeat stage. Finite element analysis performed by LLNL engineers was central in the design process to guide decisions and quantify errors. Ultratech engineers developed the design from concept to detail drawings, while in parallel, a finite element model of the complete system was developed to show in a relative sense where to make improvements to the evolving design.

\section{Background}

In the photolithographic process, microcircuit patterns are etched into subsequent layers of material deposited onto a semiconductor wafer. These overlays form a three dimensional product from an essentially two dimensional process. The stepping machine positions the wafer underneath its projection system where it casts an image (or pattern of light) of the circuit onto the layer to be etched. The process must repeat many times because the field of the lens is much smaller than the wafer being processed. The exposed areas of the material can then be removed by chemical treatments. The wafer may go on to other processes, which may include visiting other stepping machines.

The lens produces the image by focusing light that passes from an illuminator system through the mask. To provide registration, targets projected from the mask using a low intensity light source are aligned to features already existing on the wafer. The number of targets used for alignment is a compromise between the time taken to align and the reduction of systematic errors. Typically five to ten targets are used over the wafer to define its position.

\section{General Description of the Stepper}

The several major subsystems that form the metrology loop of the stepper, must each remain stable and in precise relationship to obtain good overlay accuracy. The machine base provides the rigid and stable platform necessary to support the subsystems and to maintain their relationships. The wafer motion stage mounts directly to the base, and it provides 
large scale translations of the wafer in the $x-y$ (horizontal) plane and small scale motion in the remaining four degrees of freedom for alignment purposes. Laser interferometers operating in air determine the incremental position of the stage relative to the base for $x-y$ translations and $x-y-z$ rotations. The lens also mounts directly to the base and requires a one-time adjustment to insure parallelism between the image plane and the travel of the stage. The reticle support system mounts directly to the lens. Through these subsystems, one may trace the metrology loop of critical components that include optical paths, sensors, control systems, mechanical parts and connections. The metrology loop for this stepper is somewhat unusual because the path passes twice through the lens. ${ }^{3}$

\section{System Requirements}

The lens consists of one slightly aspheric primary mirror ( $460 \mathrm{~mm}$ OD, $103 \mathrm{~mm}$ thickness, $900 \mathrm{~mm}$ concave radius) and a compact (front) assembly of transmissive optical elements. The relative spacing between the mirror and the front optical assembly $(0.7 \mathrm{~m})$ requires adjusting three degrees of freedom to a resolution of 0.2 micrometer and requires short term stability to a few nanometers. The lens housing must be air tight to contain helium because compared to air its index of refraction is less sensitive to temperature changes. ${ }^{4}$ In addition, any nonaxisymmetric deformation of the primary mirror due to gravity or parasitic forces from its supports was specified to be within a few nanometers over its lifetime. 5

The optical design of the lens required that the optical axis be inclined $15^{\circ}$ from the horizontal. The stringent deformation specification lead to the conclusion that the mirror should be inspected in the same $15^{\circ}$ orientation using a mounting arrangement identical to that in the lens. Assuming this as a requirement and the predominantly used final figuring process being polishing, then a further requirement is the capability of the mirror to be disassembled from its mounts perhaps several times. This requirement precludes any permanent potting method like that used on the front optic assembly.

The size and weight of the lens were not specifically constrained other than to fit on the stepper, but certainly there were compelling reasons to keep both as small as practical. Although a concern, cost did not appear to be a constraint as Ultratech would always choose better performance or less risk over less cost. A very important requirement for the stepper is robustness to shipping and handling. Components were designed to survive a 20 $\mathrm{g}$ acceleration to simulate an extreme shock load. Although not specifically a requirement of the stepper, microelectronics fabricators recognize the importance of and thus provide temperature controlled environments. In addition, steppers must operate in accordance to strict clean room standards.

\footnotetext{
${ }^{3}$ The first path is the mechanical connection from the base through the lens housing and to the reticle support. The second path is the optical connection from the reticle through the lens optics and to the wafer. Perhaps this indicates why the lens was the focus of this project.

${ }^{4}$ The prototype will test the need to use helium by evaluating the system using air.

${ }^{5}$ Axisymmetric deformations, if known in advance, are easy to correct in the prescription of the optic.
} 


\section{Design Overview}

The Ultratech engineering staff has a high level of competence in precision engineering as demonstrated by the design strategies applied to their stepping machines. For example, thermal expansion errors are managed by using low expansion materials such as Zerodur for the primary mirror and Invar 36 for spacing rods, by isolating heat sources and by transporting heat away from sensitive components. To manage dynamic errors, the base of the stepper is an iron casting that has closed box sections for excellent stiffness and stability. Air isolators support the stepper at four points, but two are plumbed together to form a virtual point giving three effective support points. In addition to active pneumatic level control, translations in $\mathrm{x}-\mathrm{y}$ (horizontal) and rotation about $\mathrm{z}$ (vertical) are actively controlled to counteract stage reaction forces that occur during stepping. These strategies remain unchanged for the new stepper.

Changes made to the new stepper were not so much departures from previous strategies, but instead resulted from a greater commitment to meet the requirements and from greater confidence in the designs through analysis. The commitment to analyze a design places on it an additional requirement to eliminate as far as possible those aspects that are uncertain and difficult to model; moreover, this is just good design practice. Ideally, sensitive structures such as the primary mirror should mount through a system of friction-free flexures in an exact-constraint arrangement to accommodate small motions due, for example, to differences in thermal expansion. Although this may seem at odds with the desire to maintain high resonance frequencies for lower susceptance to vibration sources, properly designed flexures can provide a ratio of primary to secondary stiffness greater than three orders of magnitude.

\section{Primary Mirror Support System}

The design began with the concept that a discrete number of weight bearing support points would attach to the primary mirror about its perimeter with the number, load distribution and orientation being determined through finite element analysis (FEA). The size and shape of the mirror were already specified because of the manufacturing lead time required. FEA of the mirror would show that too much deformation resulted from supports at only three points, where each provided two degrees of constraint. While it is possible to correct for this deformation by ion beam figuring or computer controlled polishing, the desire for multiple vendors (i.e., those without this technology) lead to the decision to use additional support points. It is possible to use six support points with each providing one degree of constraint as shown in Figure 1 , but the $15^{\circ}$ angle of the mirror is eminently close to a singularity. Likewise, a wiffle tree arrangement would be awkward, expensive and would have lower resonance frequencies.

The approach taken instead was to separate the two functions of the support system into independent mechanisms. The first provides rigid location through an exact-constraint system of flexures, while the second provides flexible weight relief at an arbitrary number of points. It would turn out that a set of nine diaphragm actuators spaced equally around the primary and oriented opposite to gravity would meet the deformation requirements using a single air regulator. This approach allows for an easy change to corrective figuring simply 
by eliminating the weight-relief mechanisms and thereby reducing costs and uncertainties in the system. Figure 2 depicts these two mechanisms.
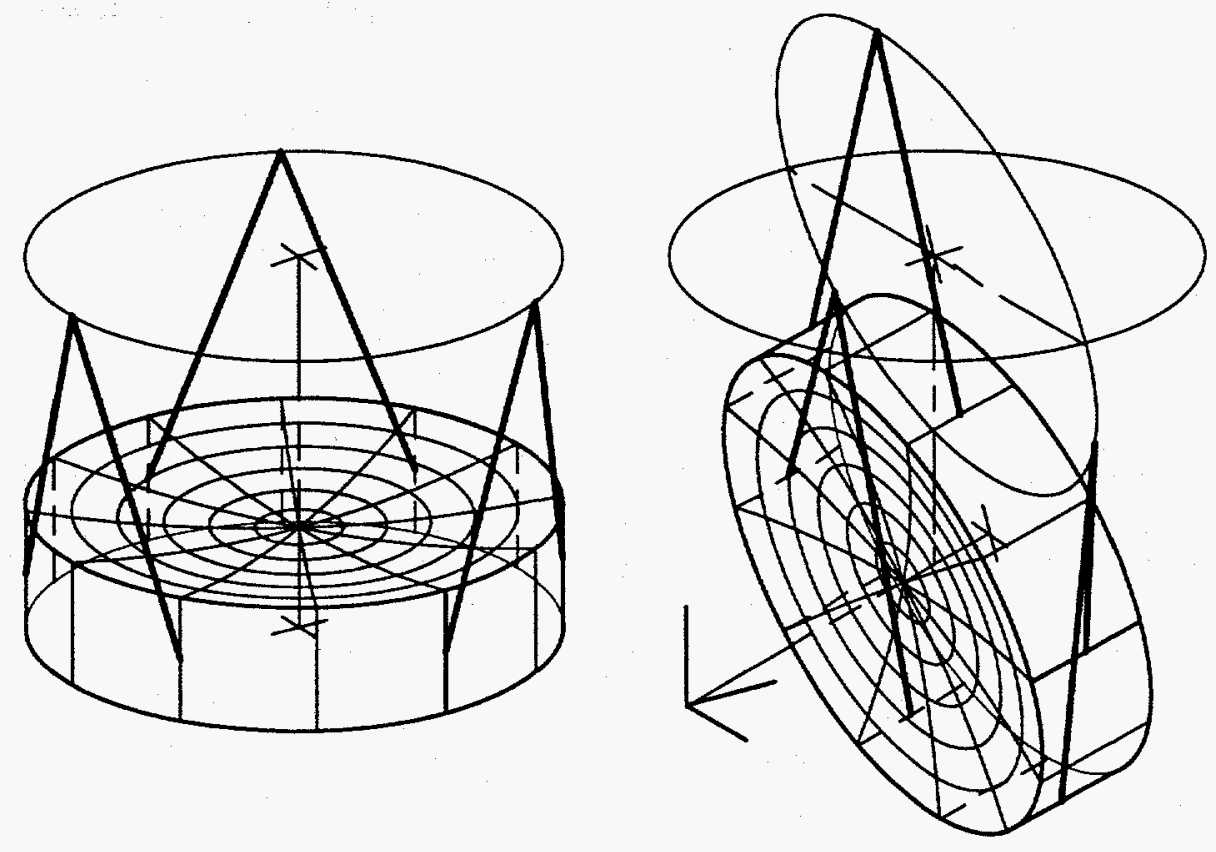

Figure 1: A six point exact-constraint mounting provides good weight distribution but approaches a singularity as the optical axis nears the horizontal.
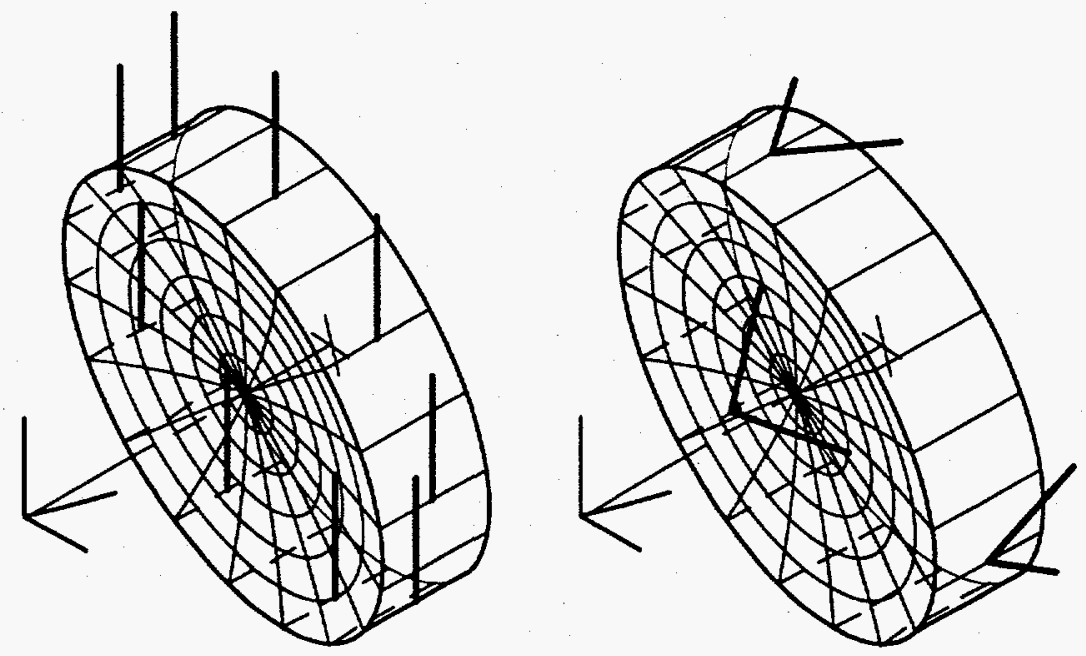

Figure 2: Nine equal forces oriented opposite to gravity provide weight relief (left), and three two-degree constraints determine the location (right).

Completing the primary mirror support system is an annular steel cell, shown in Figure 3, that provides structural support for the flexures and weight-relief mechanisms. The mirror cell, being intermediate between the mirror and the rest of the lens, provides an additional layer of protection from outside influences tending to distort the mirror. This is of particular value to the optics vendor who will use the same or similar support system during metrology for final figuring. It is interesting that the advantages of a layered approach, 
namely attenuation of disturbances, tend to be multiplicative while the disadvantages (cost, size, weight, lower frequencies) tend to be additive.

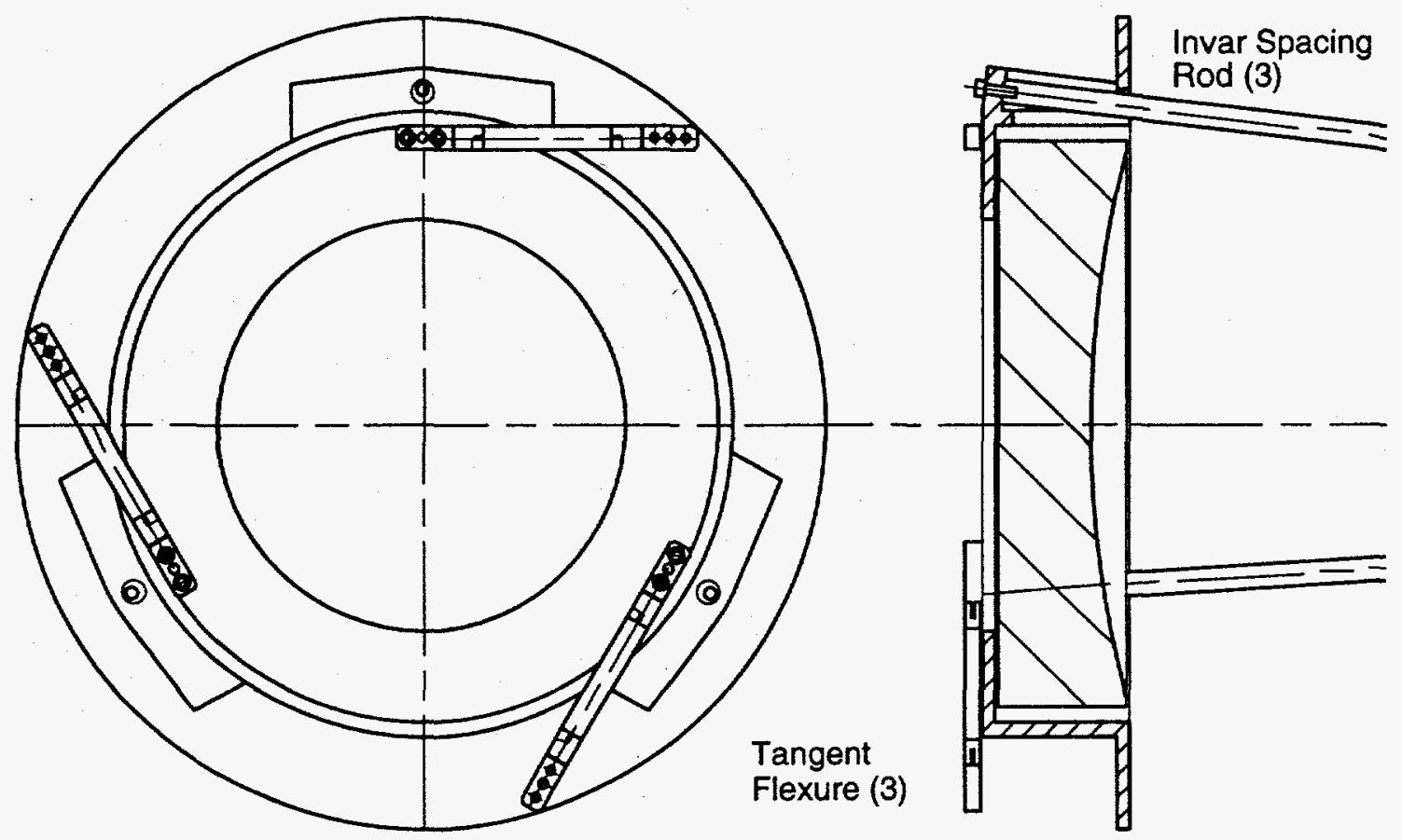

Figure 3: Three Invar spacing rods and three tangent flexures exactly constrain the primary mirror cell.

\section{Front Optics Support System}

A second annular steel cell supports the front optical assembly through 8 patches of semicompliant urethane adhesive spaced equally around its perimeter and in a plane through the optic's center of gravity. Although this is an over-constrained design, the replication process used by Ultratech provides a nearly ideal elastic averaging condition. FEA was not applied to the front optical assembly because transmissive optics are less sensitive to distortion than reflective optics and because the aspect ratio was favorable.

The front cell bolts rigidly to the front bulkhead of the lens housing in three local areas. The three pads on the cell are lapped flat and the bulkhead is ground flat to minimize forced congruence. Radial compliance at each pad was not provided because the housing and the cell are both steel. Had the housing been an aluminum casting, then radial motion flexures manufactured into the cell by wire EDM (electric discard machining) may have been necessary.

\section{Primary Mirror Adjustment System}

The mirror cell attaches to the lens housing through three Invar spacing rods (approximately $0.7 \mathrm{~m}$ long) and three flexural links as shown in Figure 3 . The spacing rods each connect to the front bulkhead through a simple collet clamp designed to allow adjustment using a differential micrometer. Together, the three adjustment mechanisms allow the focus of the primary mirror to be aligned in $x-y-z$ space relative to the front optic 
assembly, after which the micrometers are removed. Pneumatic control of axial strain in each spacing rod provides even finer resolution.

Ideally, the three spacing rods would converge to the focus of the primary mirror so that the remaining constraints were optically insensitive. Since this was not practical, the mirror is sensitive to $x-y$ motion ( $z$ being along the optical axis) relative to the front optics. To minimize the possibility of $x-y$ motion, due for example to thermal expansion, the three flexural links connect tangent to the mirror cell and the lens housing is axisymmetric to the greatest practical extent.

\section{Lens Housing}

The lens housing, shown schematically in Figure 4, is a conical steel weldment consisting of a 3/16 inch outer skin, 1/4 inch inner annular ribs, and a 3/4 inch thick front bulkhead. As mentioned previously, the front cell, the reticle stage and the spacing rods all attach to the front bulkhead making it a critical component of the housing. The rear of the housing is open to permit installation of the mirror cell. A $1 / 2$ inch thick cover plate bolts to a rear flange on the housing making it structurally closed. The three tangent-link flexures attach to the cover plate, and together with the mirror cell, install conveniently as a subassembly.

\section{Adjustable Exact-Constraint Mounting}

Six adjustable-length rods each connect the lens housing to the base of the stepper. Both ends of each rod thread into ball joints to form a single-constraint turn buckle. ${ }^{6} \mathrm{~A}$ linear transformation exists (assuming small motions) between adjustments made at the rods and the resulting motion of the lens, and the transformation matrix is called the Jacobian. More useful is the inverse transformation given by $\mathrm{T}$ in Figure 4, which is determined from the nominal locations of the rods (identified by $i=1 . .6$ ) given by the vectors $(x, y, z, \alpha, \beta)_{i}$. Columns of $T$ taken left to right represent the required rod adjustments $(1 . .6)$ for pure translations in $x-y-z$, respectively, and for pure rotations in $x-y-z$, respectively.

An important feature of the adjustable-length rod shown in Figure 5 is the ability to provide stiff, backlash-free constraint along its axis and flexural freedom in all other directions. Each sphere rests in a gothic arch shaped socket to provide a thin ring of contact. A similarly shaped clamping nut preloads the ball into the socket, and by virtue of the flexural cuts, also preloads the threads. Adjustments are made prior to tightening all of the nuts so that the balls are free to pivot and the rods are free to rotate.

\footnotetext{
${ }^{6}$ Opposite hand threads were used in this case to provide greater travel of adjustment. Different lead threads would instead provide finer resolution of travel.
} 

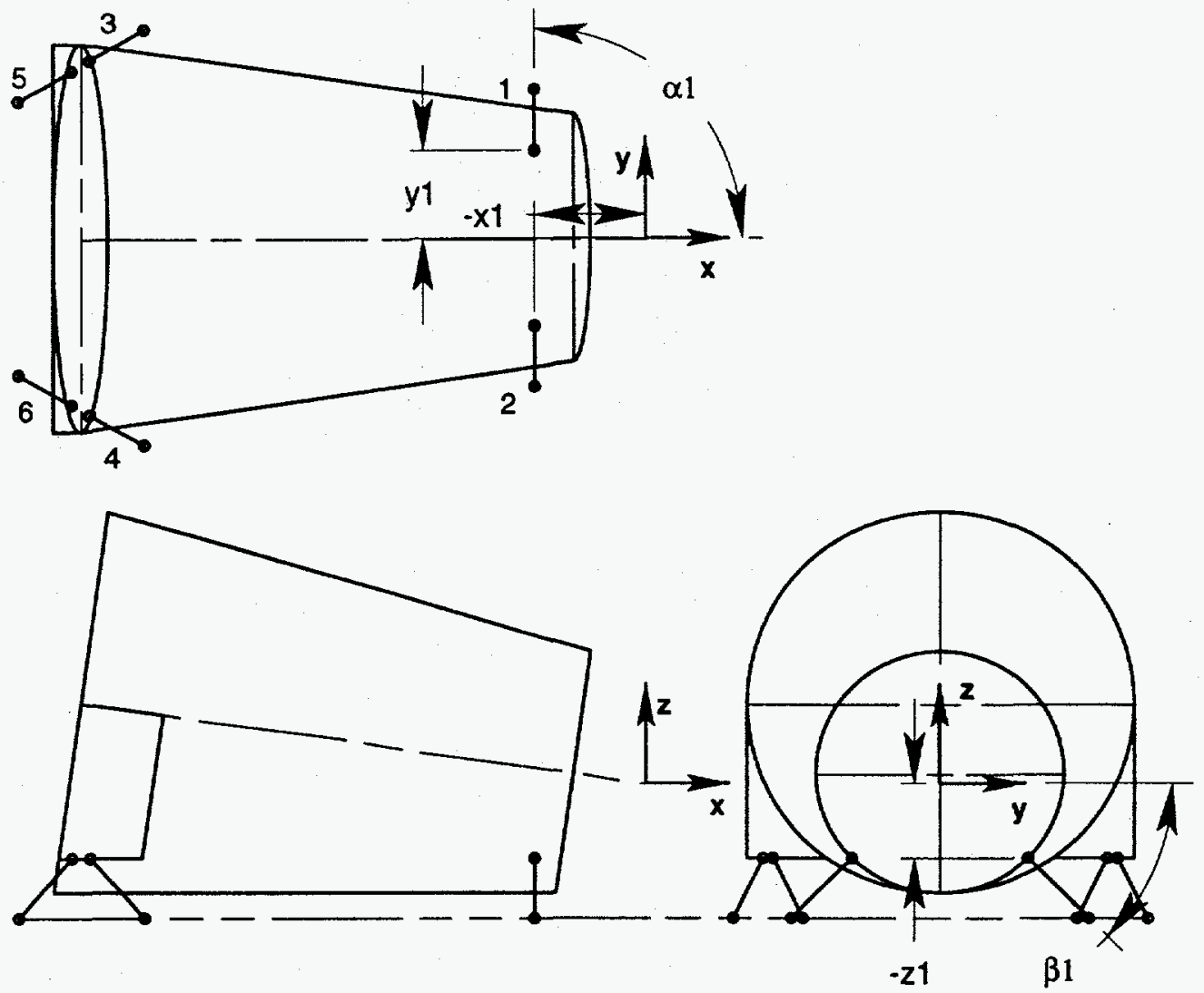

$\left[\begin{array}{l}x_{i} \\ y_{i} \\ z_{i} \\ \alpha_{i} \\ \beta_{i}\end{array}\right]:=\left[\begin{array}{cccccc}-11.18 & -11.18 & -31.81 & -31.81 & -33.03 & -33.03 \\ 7.45 & -7.45 & 10.66 & -10.66 & 9.41 & -9.41 \\ -2.20 & -2.20 & -2.20 & -2.20 & -2.20 & -2.20 \\ \frac{\pi}{2} & -\frac{\pi}{2} & \frac{\pi}{6} & -\frac{\pi}{6} & \frac{7 \cdot \pi}{6} & -\frac{7 \cdot \pi}{6} \\ \frac{\pi}{4} & \frac{\pi}{4} & \frac{\pi}{4} & \frac{\pi}{4} & \frac{\pi}{4} & \frac{\pi}{4}\end{array}\right]$

$$
\mathrm{T}=\left[\begin{array}{lccccc}
0 & 0.707 & 0.707 & 6.824 & 7.905 & -7.905 \\
0 & -0.707 & 0.707 & -6.824 & 7.905 & 7.905 \\
0.612 & 0.354 & 0.707 & 8.316 & 21.146 & -17.774 \\
0.612 & -0.354 & 0.707 & -8.316 & 21.146 & 17.774 \\
-0.612 & -0.354 & 0.707 & 5.876 & 24.703 & 17.44 \\
-0.612 & 0.354 & 0.707 & -5.876 & 24.703 & -17.44
\end{array}\right]
$$

Figure 4: The transformation matrix $T$ indicates the adjustments required at rods $1 . .6$ to move the lens relative to the $x-y-z$ coordinate system as defined above. 


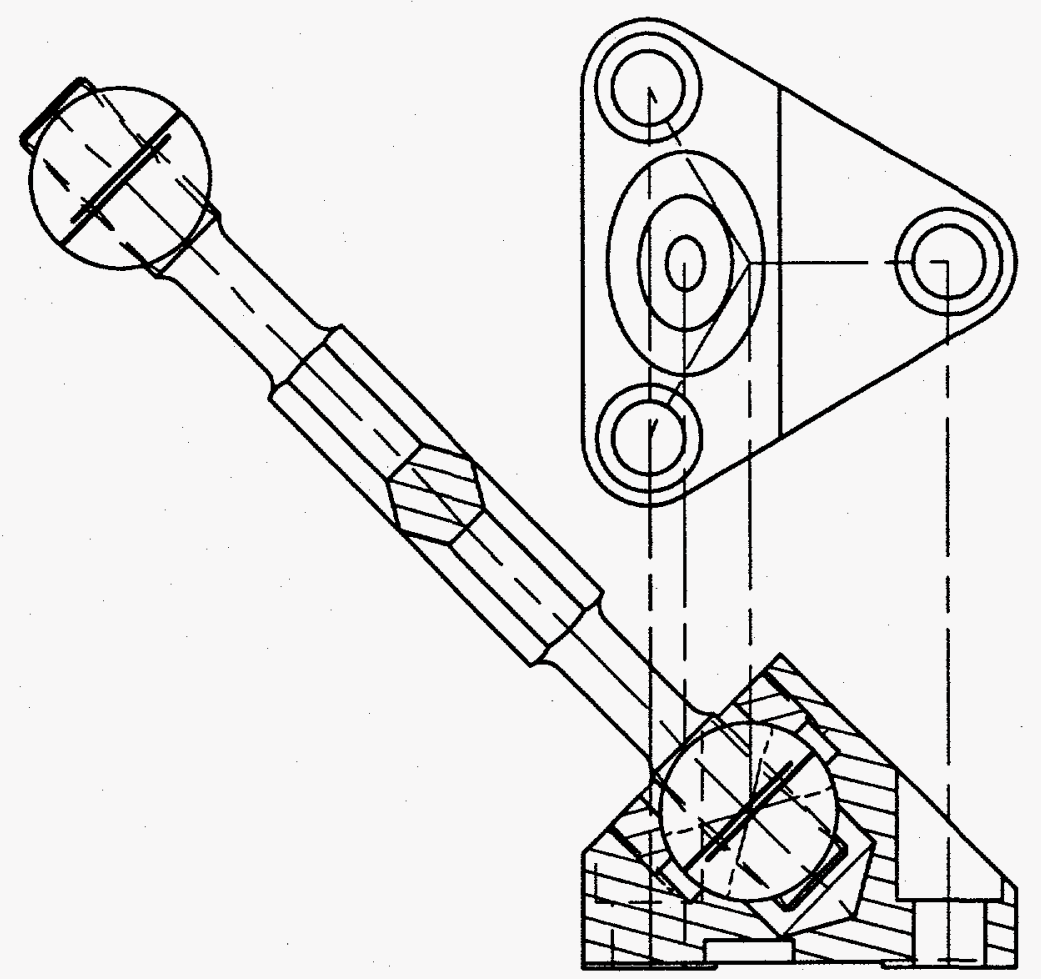

Figure 5: The clamping nut simultaneously preloads the ball socket and the threads making it a stiff, backlash-free joint.

\section{Deformation Analysis of the Primary Mirror}

Various mounting configurations of the primary mirror were analyzed for gravity induced deformation to arrive at an acceptable design. These included uniform perimeter support and distributed perimeter supports at three, six and nine points. To more easily develop an intuitive understanding for the results, the actual gravity vector was separated into orthogonal components as shown in Figure 6, and the two load cases were solved independently. By the principle of superposition, the actual solution is equal to the sum of the independent solutions. Numerical checks did verify the approach.

Invariably, an optic's spherical component of deformation will be very small compared to the tolerance on its basic radius of curvature. This allows that component to be counted as an insignificant tolerance rather than a significant deformation. Further, any axisymmetric, aspheric deformation, if known in advance, can be compensated in the prescription of the optic. These points are inconsequencial to the physical optic given the current manufacturing plan, but rather they ease the design requirements of the support system. Thus all design decisions were based on the nonaxisymmetric portion of the deformed shape after the best-fit sphere was removed.

All deformation analyses on the primary mirror, with the exception of an early closed-form plate model, were modeled using a commercial finite element program (Applied Structures by Rasna Corp.). The nominal spherical shape of the mirror was modeled with solid elements, and the deflections of the surface points were post-processed in a commercial mathematics program (Matlab by the Math Works Inc.) to remove the 
spherical component and to generate 3D surface plots. The degree of asphericity in the mirror is insignificant to the mechanics of the model.

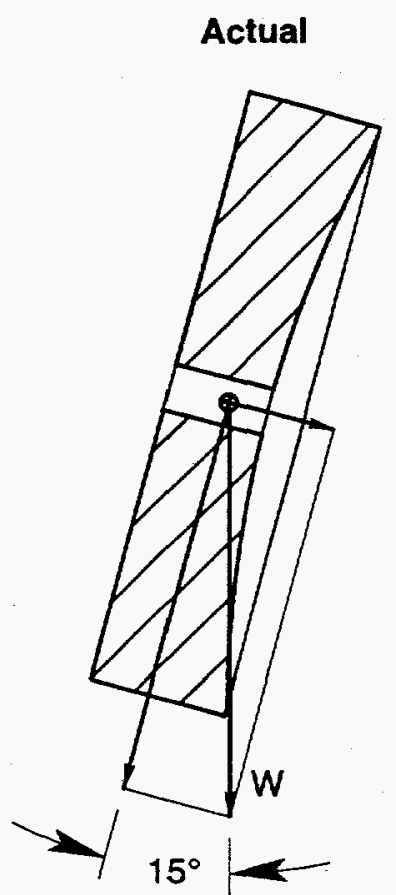

Z Gravity

X Gravity

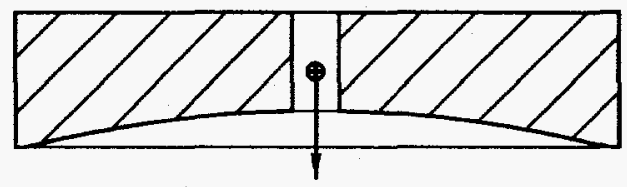

$W \sin 15^{\circ}$

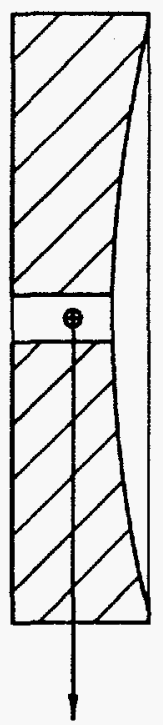

$W \cos 15^{\circ}$

Figure 6: The deformation of the mirror in its actual $15^{\circ}$ orientation is equal to the superposition of two deformations due to $\mathrm{X}$ and $\mathrm{Z}$ components of gravity.

\section{Removing the Spherical Component of Deformation}

Equation 1 represents any point $i$ on the deformed and displaced surface of the initially spherical mirror. The goal is to remove the spherical component obtained by globally minimizing the spherical error at sampled points represented by $e_{i}$. The nominal sphere represented by $x, y, z$, and $r$, does not change through the fitting process. The spherical deformation represented by $d r$, and the displacement of the center represented by $a, b$, and $c$, are small enough compared to the nominal to allow a linear analysis.

$$
\begin{aligned}
\left(x_{i}+\right. & \left.d x_{i}-a\right)^{2}+\left(y_{i}+d y_{i}-b\right)^{2}+\left(z_{i}+d z_{i}-c\right)^{2}=\left(r+d r+e_{i}\right)^{2} \\
& x_{i}^{2}+y_{i}^{2}+z_{i}^{2}-r^{2} \\
& +\left(d x_{i}-a\right)^{2}+\left(d y_{i}-b\right)^{2}+\left(d z_{i}-c\right)^{2}-\left(d r+e_{i}\right)^{2} \\
& +x_{i}\left(d x_{i}-a\right)+y_{i}\left(d y_{i}-b\right)+z_{i}\left(d z_{i}-c\right)-r d r=r e_{i}
\end{aligned}
$$

The first step in the derivation is to expand the squares of Equation 1 and to segregate terms as shown by Equation 2. The first line in Equation 2 is the nominal sphere, which is equal to zero as written. The second line consists only of squares of small relative terms that are negligible. The third line contains all significant information from Equation 1 but in a linear form. With this the deflections at all surface points in the analysis are put into a matrix form that is convenient for a least-squares analysis. In Equation 3, $\mathbf{A}$ represents 
the nominal sphere, which is constant from analysis to analysis, $\mathbf{x}$ represents the fitted sphere to be found, $\mathbf{b}$ contains the calculated deflections, and $\mathbf{e}$ is the error vector to be minimized.

$$
\begin{array}{r}
{\left[\begin{array}{cccc}
x_{1} & y_{1} & z_{1} & -r \\
x_{2} & y_{2} & z_{2} & -r \\
\vdots & \vdots & \vdots & \vdots \\
x_{n} & y_{n} & z_{n} & -r
\end{array}\right] \cdot\left[\begin{array}{c}
a \\
b \\
c \\
d r
\end{array}\right]-\left[\begin{array}{c}
x_{1} d x_{1}+y_{1} d y_{1}+z_{1} d z_{1} \\
x_{2} d x_{2}+y_{2} d y_{2}+z_{2} d z_{2} \\
\vdots \\
x_{n} d x_{n}+y_{n} d y_{n}+z_{n} d z_{n}
\end{array}\right]=r\left[\begin{array}{c}
e_{1} \\
e_{2} \\
\vdots \\
e_{n}
\end{array}\right]} \\
\mathbf{A} \cdot \mathbf{x}-\mathbf{b}=r \mathbf{e}
\end{array}
$$

The least-squares method minimizes the sum of squared errors or equivalently the length of the error vector. This assumes that each component (or point) carries the same importance to the total. Otherwise a (normally) diagonal weighting matrix that represents the relative importance of components, must multiply the error vector. The weighting matrix $\mathbf{W}$ in Equation 4 has diagonal entries in proportional to the area represented by the corresponding points. The length of the weighted error vector is minimized in Equations 4 and 5 by setting its gradient to zero and solving for $\mathbf{x}$.

$$
\begin{gathered}
\frac{d}{d \mathbf{x}}\left[\|\mathbf{W}(\mathbf{A} \cdot \mathbf{x}-\mathbf{b})\|^{2}\right]=\left(\mathbf{A}^{\mathrm{T}} \mathbf{W}^{\mathrm{T}} \mathbf{W} \mathbf{A}\right) \mathbf{x}-\mathbf{A}^{\mathrm{T}} \mathbf{W}^{\mathrm{T}} \mathbf{W} \cdot \mathbf{b}=\mathbf{0} \\
\mathbf{x}=\left(\mathbf{A}^{\mathrm{T}} \mathbf{W}^{\mathrm{T}} \mathbf{W} \mathbf{A}\right)^{-1} \mathbf{A}^{\mathrm{T}} \mathbf{W}^{\mathrm{T}} \mathbf{W} \cdot \mathbf{b}
\end{gathered}
$$

It was interesting and perhaps obvious that a sphere fitted to the combined gravity load was the simple sum of spheres fitted to the $\mathrm{X}$ and $\mathrm{Z}$ components of gravity, while the rms errors combined by vector addition.

\section{Uniform Perimeter Support}

When limited to only perimeter support, a uniform distribution of force provides the primary mirror with the best possible support to yield minimum residual error (i.e., the result after removing the spherical deformation). The Z-gravity load case produces purely axisymmetric deformation, which is inconsequencial as described previously. Therefore, uniform perimeter support represents the ideal to compare with various schemes of distributed perimeter support.

Early in the project before we could post-process FEA results, a closed-form plate model provided the first measure of the magnitudes to expect. The deflection equation for a constant thickness disk (from Roark and Young) was coded into a commercial mathematics program (Mathcad by Mathsoft Inc.). The thickness was judged to be $80 \mathrm{~mm}$, which is somewhat greater than the thinnest point on the mirror $(73.9 \mathrm{~mm})$. Figure 7 shows the deflection of the plate to a Z-gravity load plotted as a function of its radius. Also plotted is 
the fitted sphere based on area. Figure 8 shows the difference between these two curves. The residual error is $2.6 \mathrm{~nm}(\mathrm{p}-\mathrm{v})$ or $0.4 \mathrm{~nm}(\mathrm{rms}) .^{7}$

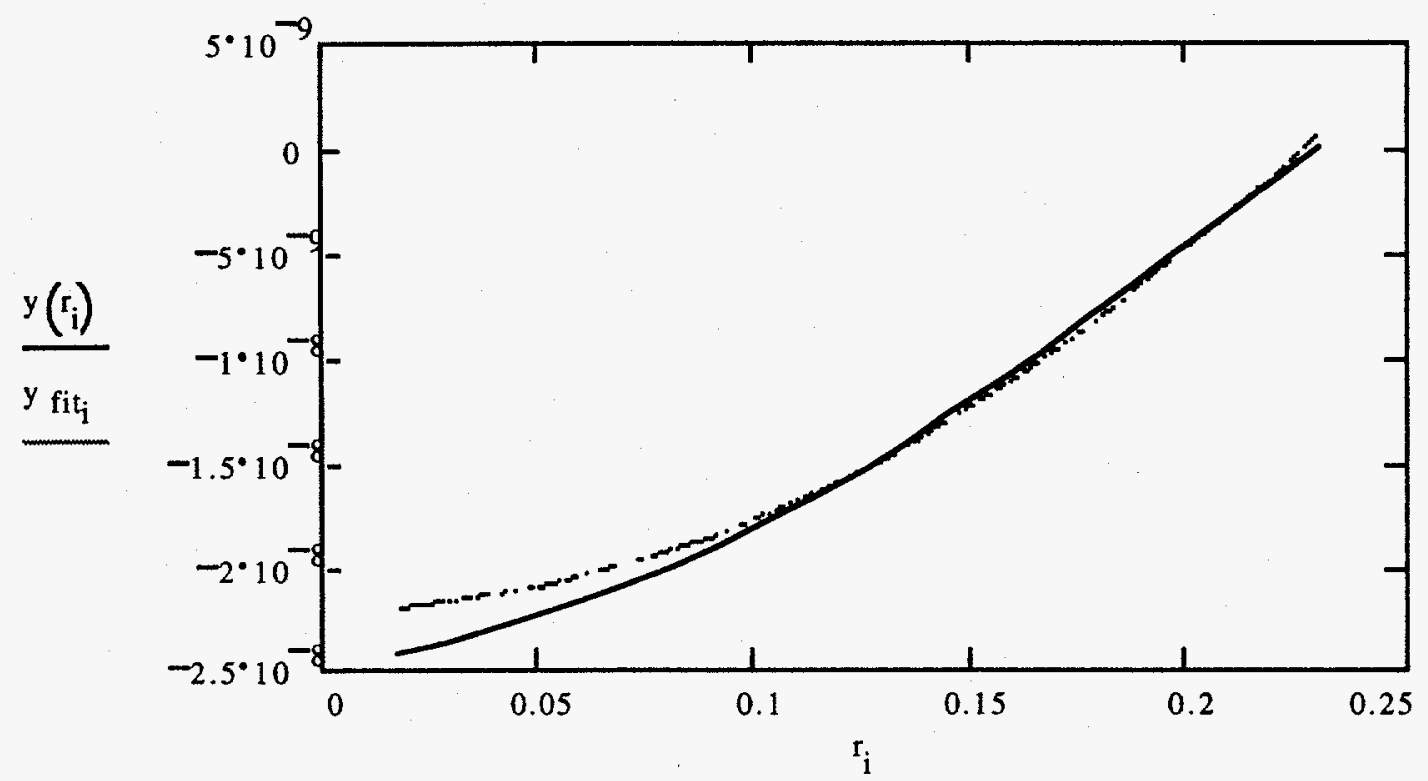

Figure 7: Deformation vs. radius (solid curve) for the $80 \mathrm{~mm}$ thick plate model and the fitted sphere based on area vs. radius (broken curve). Both axes are in meters.

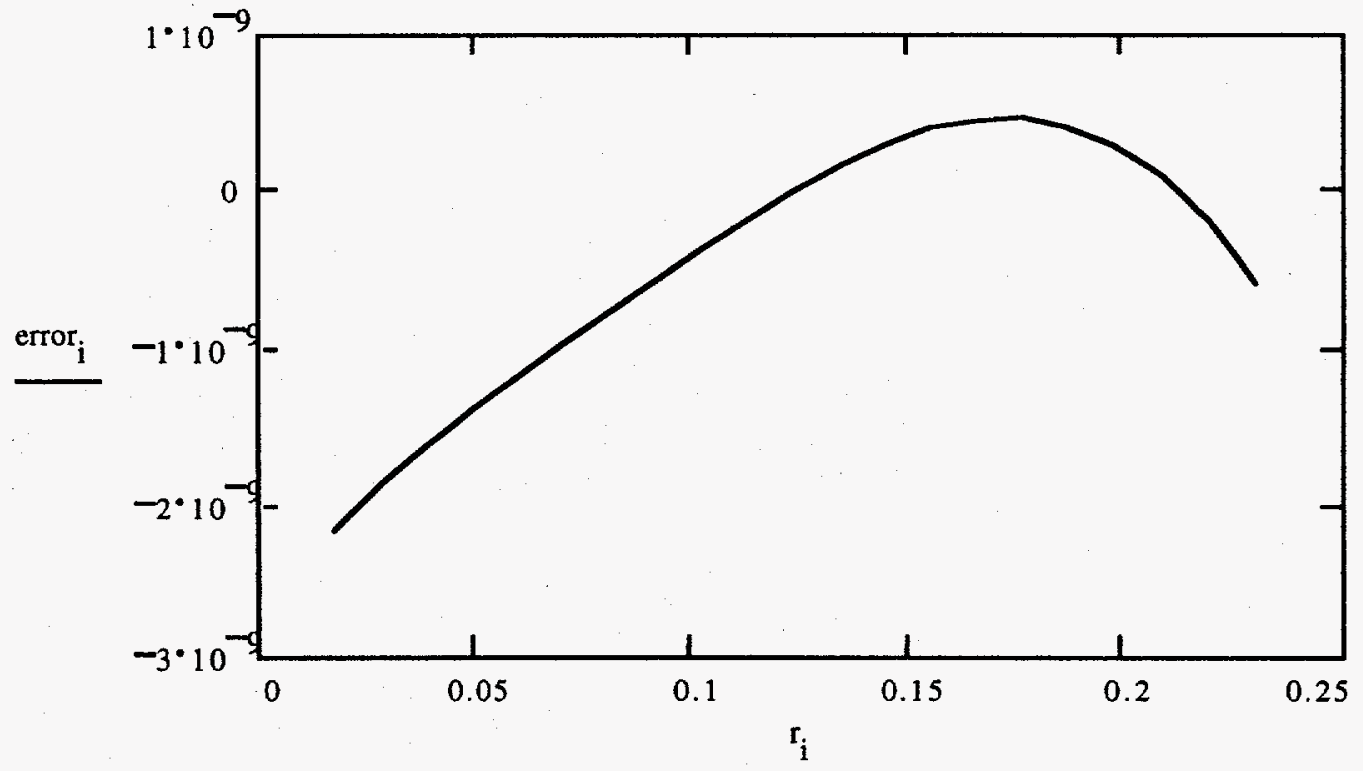

Figure 8: Residual error (difference between deformation and fitted sphere) vs. radius for the $80 \mathrm{~mm}$ thick plate model. Both axes are in meters.

The finite element model of the mirror for uniform perimeter support and Z-gravity loading predicted a slightly greater residual error of $3.9 \mathrm{~nm}(\mathrm{p}-\mathrm{v})$ or $0.5 \mathrm{~nm}$ (rms). Figure 9

${ }^{7}$ The abbreviations $\mathrm{nm}, \mathrm{p}-\mathrm{v}$ and $\mathrm{rms}$ stand for nanometer, peak-to-valley and root-mean-square, respectively. All results expressed as rms are based on area. 
shows the residual error represented as a three dimensional surface for one half of the mirror.

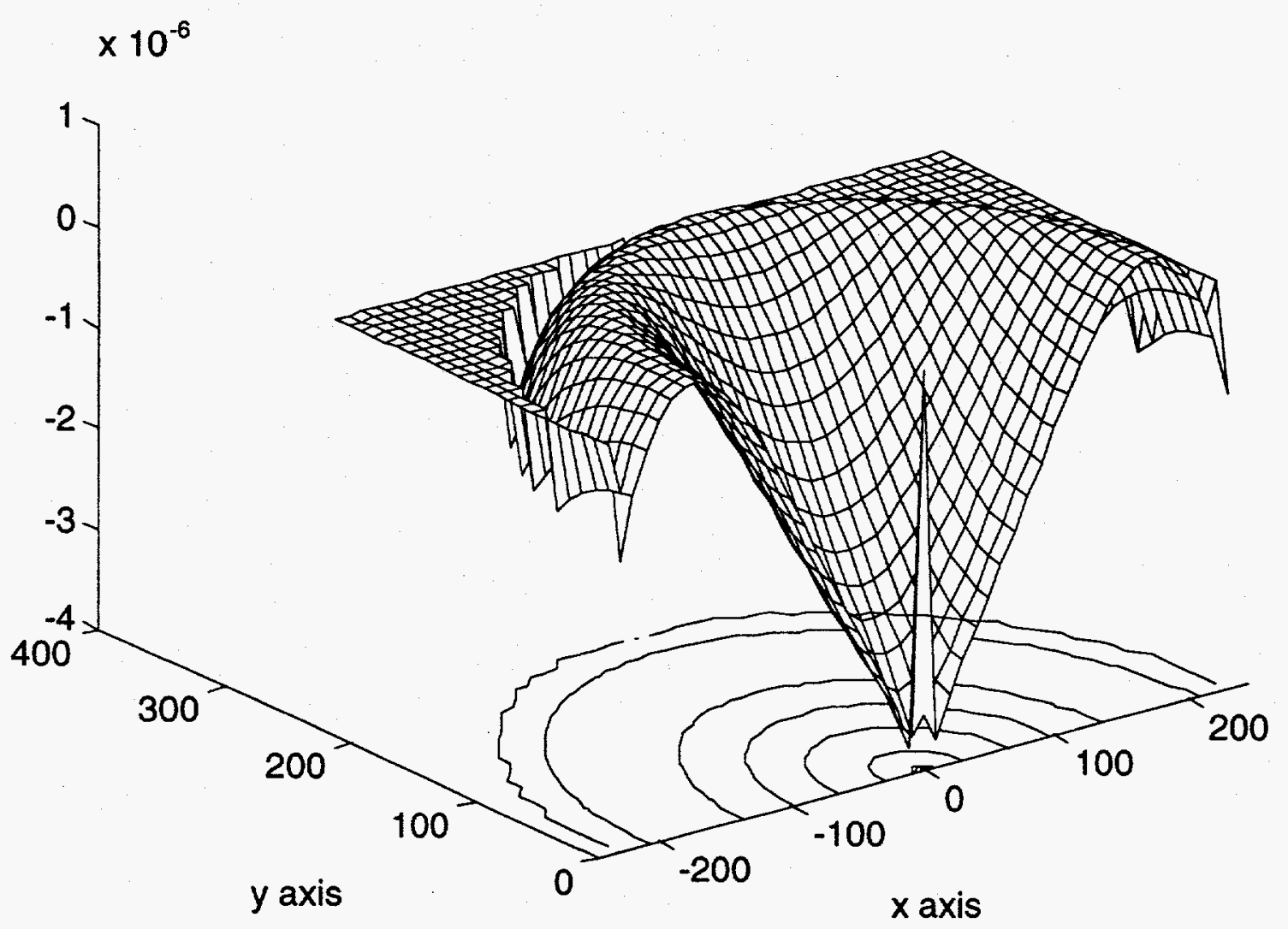

Figure 9: Surface plot of residual errors for uniform perimeter support. All axes are in millimeters. One half of the primary mirror is shown.

\section{Distributed Perimeter Support}

The goal of the analysis was to determine how to support the primary mirror such that its nonaxisymmetric deformation was less than $\lambda / 100$ or $3.85 \mathrm{~nm}(\mathrm{p}-\mathrm{v})$. Specifically, the number and location of support points around the perimeter, and the distribution and orientation of reaction forces at those points were required in order to design the support system. If uniform support is one ideal calling for a greater number of supports, then the competing ideal is the desire to keep the design simple, low cost and tractable. Thus we started with three points and worked upward until satisfying the deformation specification.

Several observations helped to guide the analysis and the design. First, the mirror is most sensitive to bending and directly affected by Z-gravity; although, $\mathrm{X}$-gravity is almost four times stronger and may be significant. Second because of axisymmetry, the Z-gravity load is best supported by an equal angular spacing of support points having equal Zreaction forces. To preserve the second point, the resultant of the X-reaction forces must pass through the center of gravity, which is most easily accomplished by placing all support points in a plane through the center of gravity. 
The first design study was of a three-point, exact-constraint support system. This is equivalent to Figure 2 without the separate weight-relief system. While all three points support Z-reaction forces, only the two side points support X-reaction forces. The residual error for the Z-gravity case, shown in Figure 10, is $35.1 \mathrm{~nm}(\mathrm{p}-\mathrm{v})$ or $7.8 \mathrm{~nm}(\mathrm{rms})$. The residual error for the X-gravity case, shown in Figure 11, is $16.3 \mathrm{~nm}(\mathrm{p}-\mathrm{v})$ or $2.2 \mathrm{~nm}$ (rms). The combined residual error is approximately $43 \mathrm{~nm}(\mathrm{p}-\mathrm{v})$ and greater than the goal by an order of magnitude, which clearly indicates the need for more supports (or final figuring) ${ }^{8}$

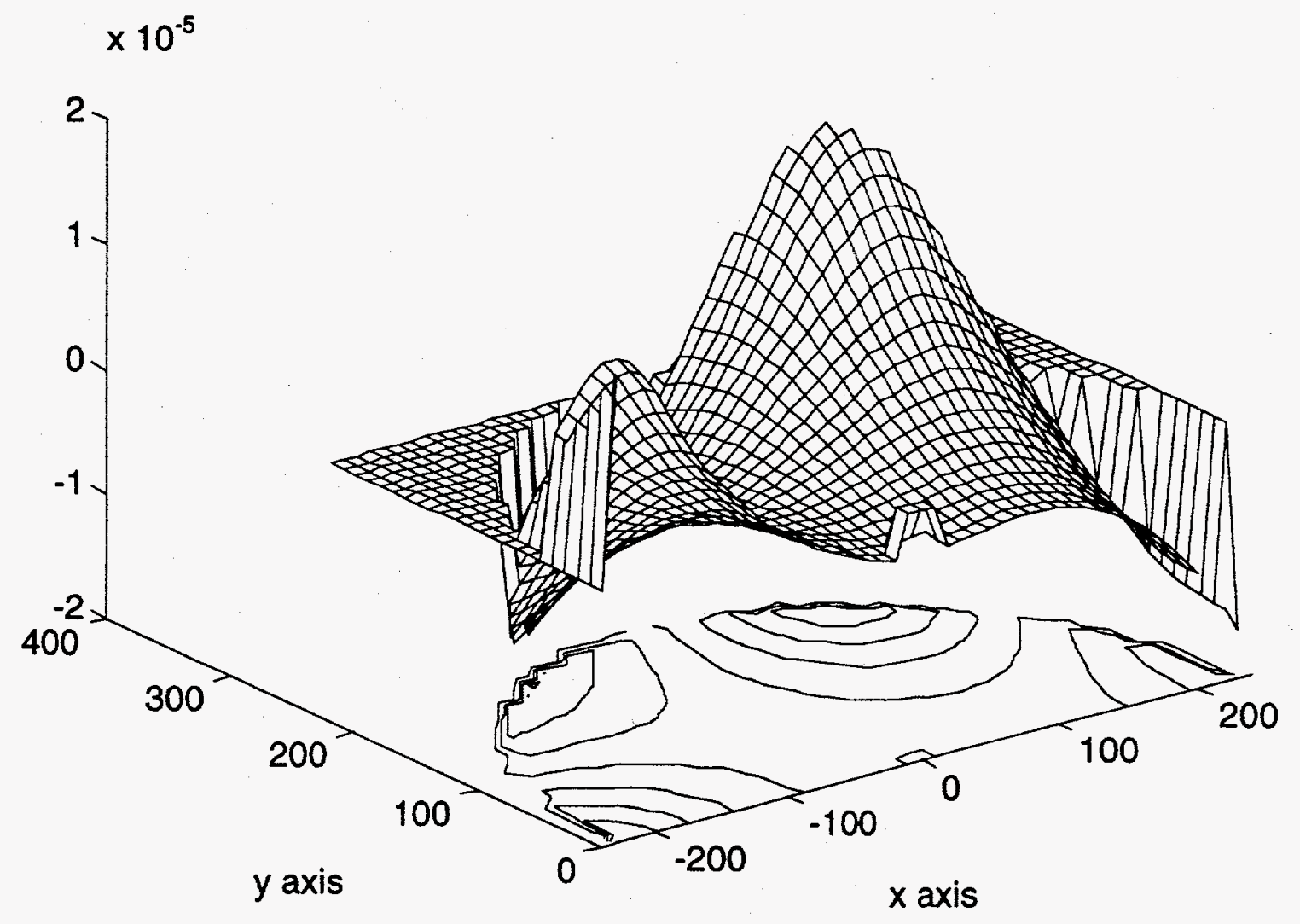

Figure 10: Residual errors for Z-gravity and three-point perimeter support. All axes are in millimeters. One half of the primary mirror is shown.

\footnotetext{
${ }^{8}$ The combined error was obtained by adding the peak from $\mathrm{X}$ to the peak-to-valley from $\mathrm{Z}$, since the peaks occur very near one another.
} 


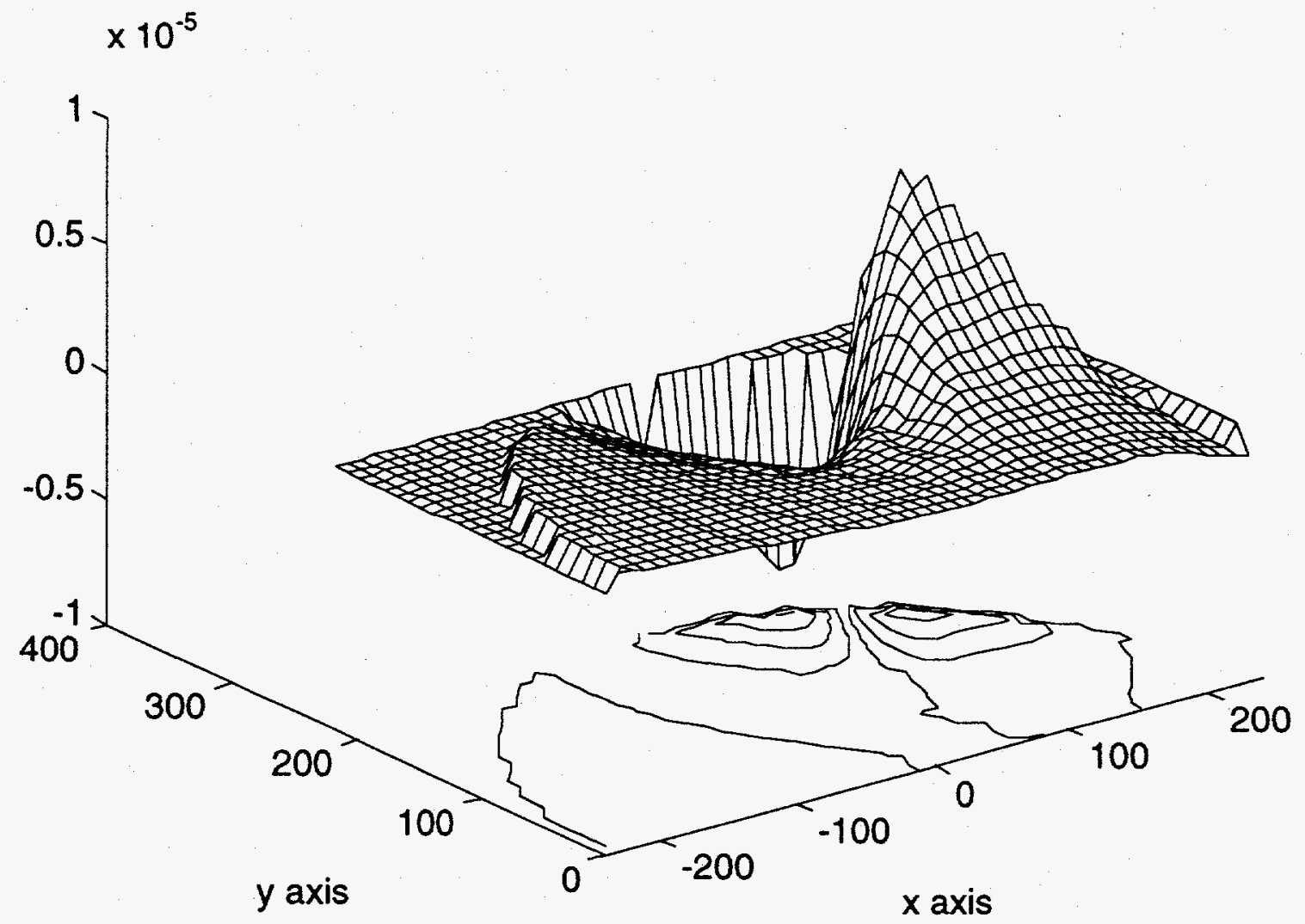

Figure 11: Residual errors for X-gravity and three-point perimeter support. All axes are in millimeters. One half of the primary mirror is shown.

The relative significance of the $\mathrm{X}$-gravity load was somewhat surprising, and the shape of the plot indicates local bending near the support due to a moment load. This occurs because the primary mirror is nonsymmetric about the $x-y$ plane (i.e., concave on one side). The reaction force does not pass through the local centroid, thereby causing a moment due to combined effects of imbalanced weight and displaced shear center. Simply moving the support trades the local problem for a larger global problem. The simple fix is to make the mirror symmetric (i.e., doubly concave), but that was not possible for the prototype machine.

In the second study, the primary mirror was over constrained at six points so that the reaction forces would be outputs rather than inputs. Figure 12 shows that the reaction forces are equal in $\mathrm{Z}$ and nearly equal in $\mathrm{X}$ as anticipated. The residual error for the $\mathrm{Z}$ gravity case, shown in Figure 13, is $5.9 \mathrm{~nm}(\mathrm{p}-\mathrm{v})$ or $1.2 \mathrm{~nm}(\mathrm{rms})$. The residual error for the X-gravity case, shown in Figure 14, is $4.9 \mathrm{~nm}(\mathrm{p}-\mathrm{v})$ or $1.0 \mathrm{~nm}(\mathrm{rms})$. The combined residual error is $9.9 \mathrm{~nm}(\mathrm{p}-\mathrm{v})$ or $1.5 \mathrm{~nm}(\mathrm{rms})$, and greater than the goal of 3.9. It appears, however, that six weight-relief points on a symmetric primary mirror would very nearly meet the goal. In that case, the nonaxisymmetric error would be approximately $5 \mathrm{~nm}(\mathrm{p}-\mathrm{v})$. 


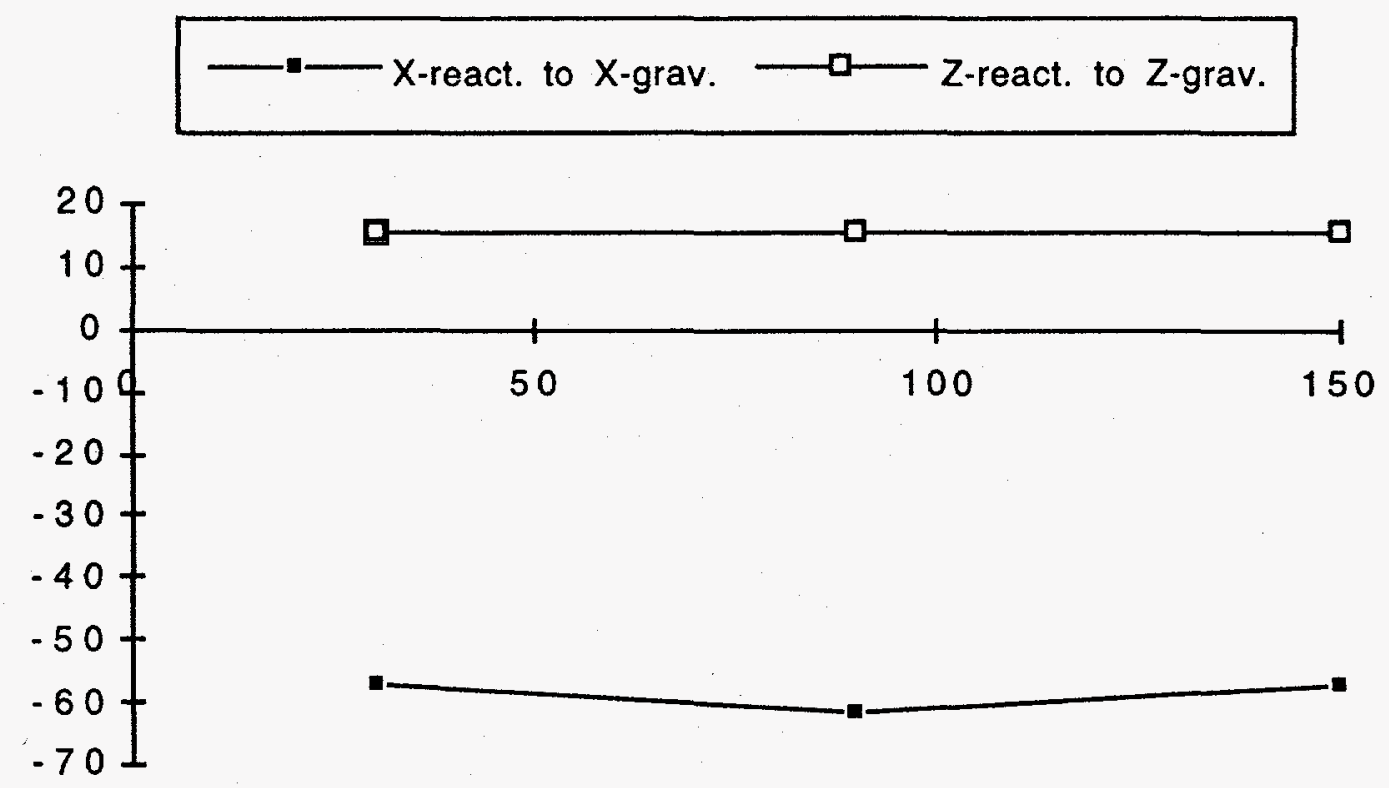

Figure 12: Reaction force (newton) vs. angle for six fixed perimeter supports.

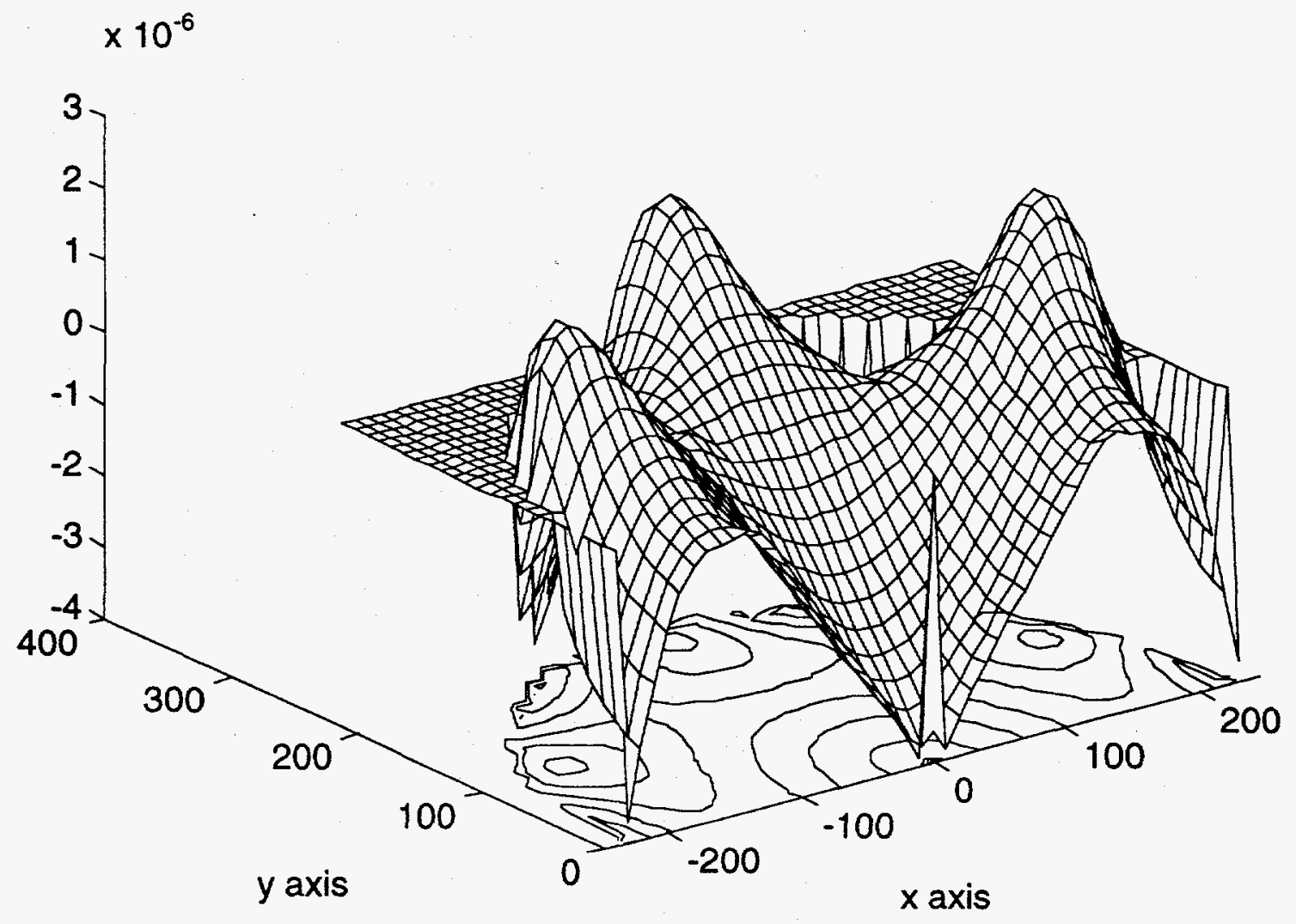

Figure 13: Residual errors for Z-gravity and six-point perimeter support. All axes are in millimeters. One half of the primary mirror is shown. 


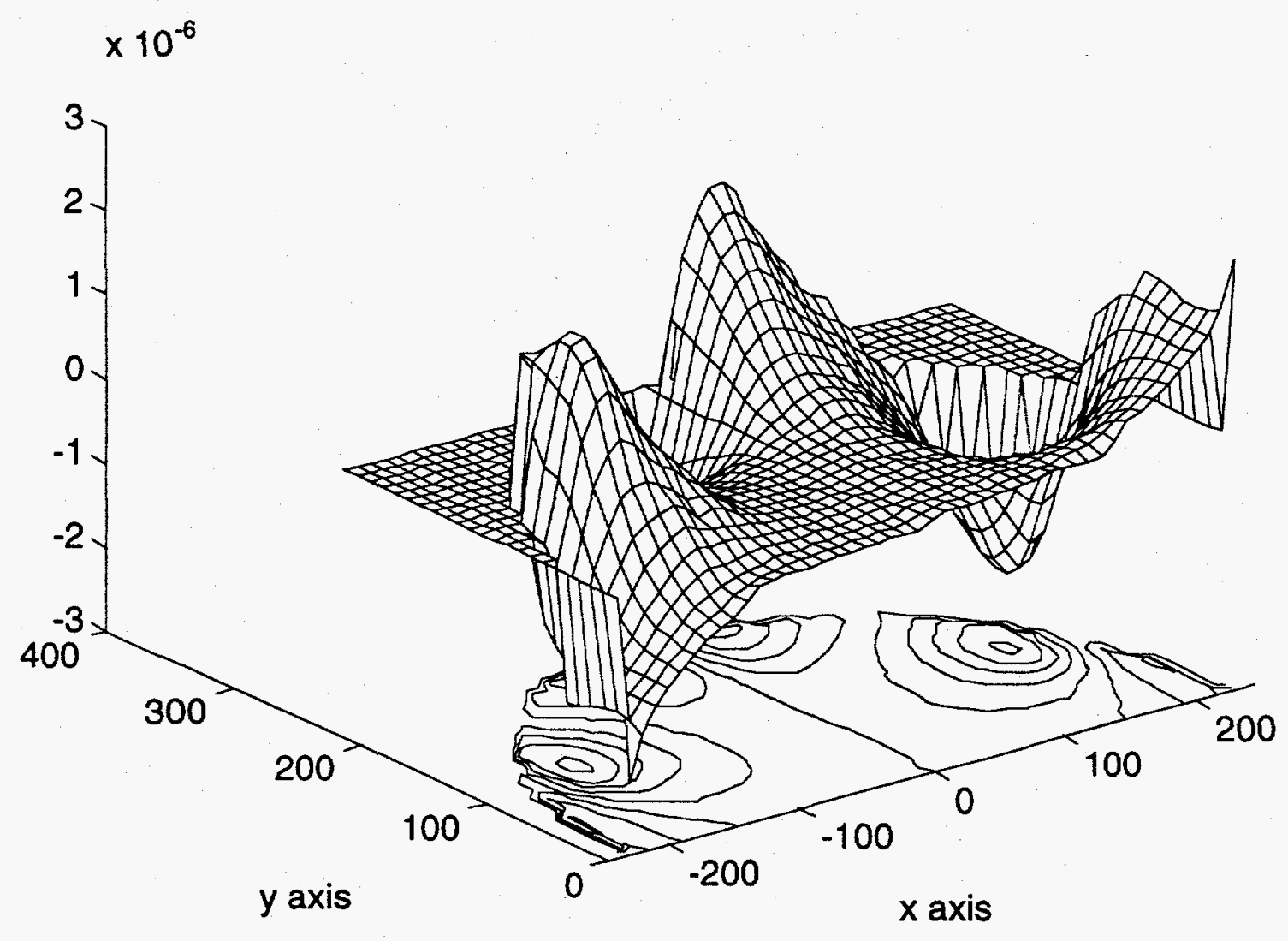

Figure 14: Residual errors for X-gravity and six-point perimeter support. All axes are in millimeters. One half of the primary mirror is shown.

In the third study, the primary mirror was over constrained at nine points, and again as shown by Figure 15, the Z-reaction forces are equal while those in $\mathrm{X}$ are not quite as equal. The analysis was repeated under the condition shown in Figure 2 to determine the deformation. The residual error for the Z-gravity case, shown in Figure 16, is $4.5 \mathrm{~nm}(\mathrm{p}-\mathrm{v})$ or $0.7 \mathrm{~nm}(\mathrm{rms})$. This includes a relatively large axisymmetric component given approximately by the uniform support case. The nonaxisymmetric error is $2.5 \mathrm{~nm}(\mathrm{p}-\mathrm{v})$ or $0.5 \mathrm{~nm}(\mathrm{rms}) .{ }^{9}$ The residual error for the X-gravity case, shown in Figure 17, is $3.0 \mathrm{~nm}$ (p-v) or $0.5 \mathrm{~nm}(\mathrm{rms})$. The combined nonaxisymmetric error is $4.2 \mathrm{~nm}(\mathrm{p}-\mathrm{v})$ or $0.7 \mathrm{~nm}$ (rms), which is satisfactory.

It is interesting at this point to summarize the effect of increasing the number of supports. For X-gravity, more supports will share the weight so that the local moment and deformation should decrease linearly. For Z-gravity, the span decreases linearly so that the deformation should decrease by a higher power. For comparison, the deflection of a uniformly loaded beam goes as the fourth power of the span. Figure 18 summarizes the results and shows that the $\mathrm{X}$-gravity load becomes increasingly more significant and is best dealt with by symmetry.

${ }^{9}$ The nonaxisymmetric peak-to-valley error was taken at the perimeter. The rms error was obtained by subtracting the uniform support case from the residual in a vector sense. 


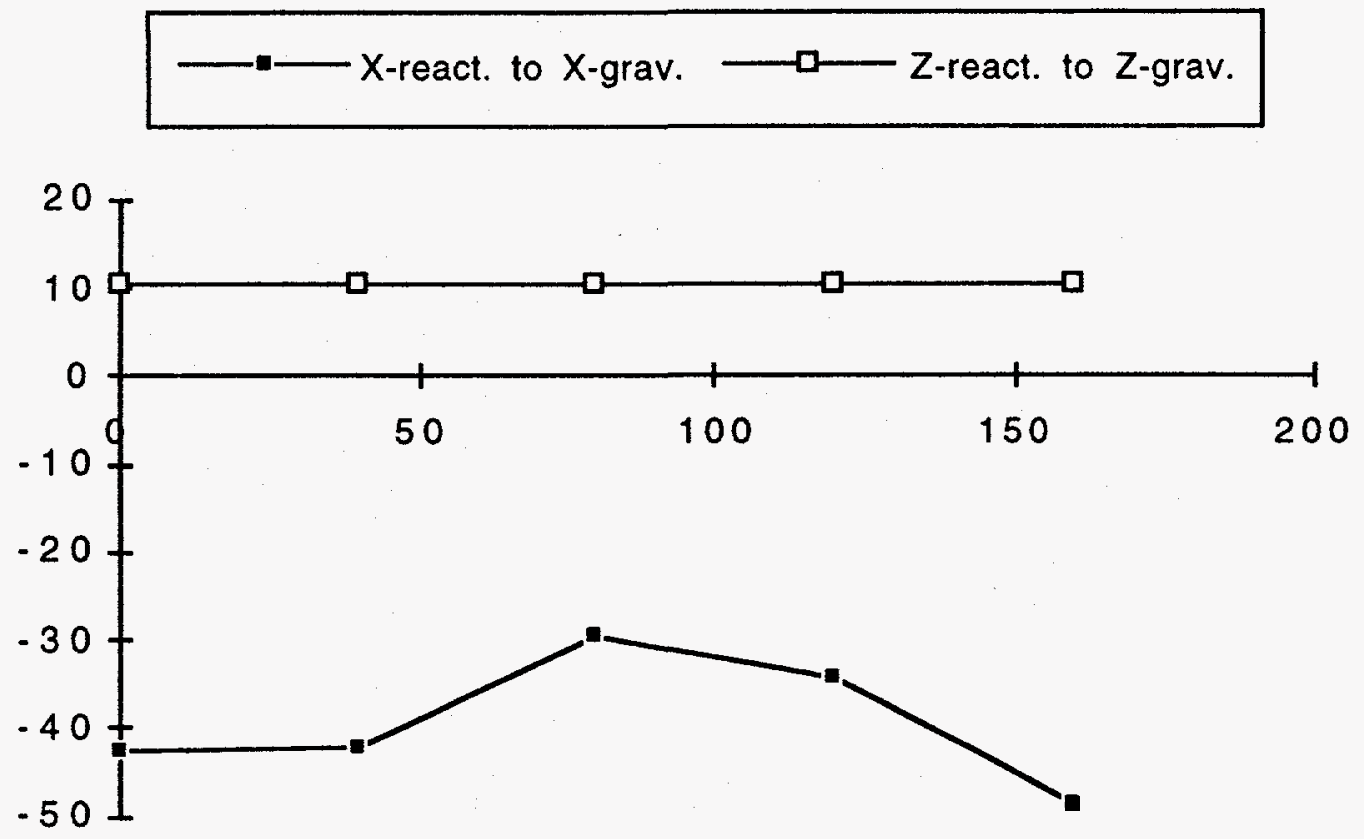

Figure 15: Reaction force (newton) vs. angle for nine fixed perimeter supports.

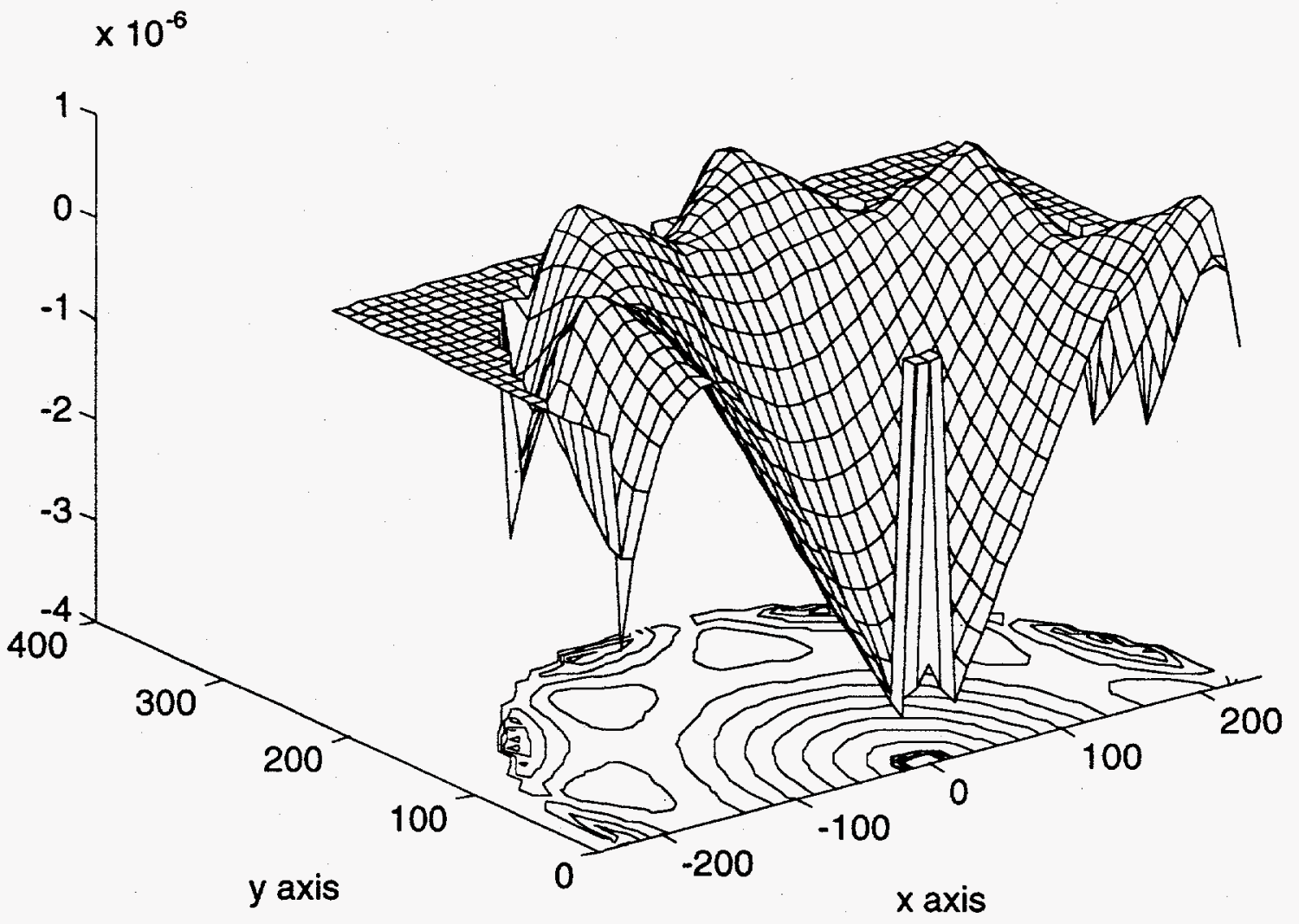

Figure 16: Residual errors for Z-gravity and nine-point perimeter support. All axes are in millimeters. One half of the primary mirror is shown. 


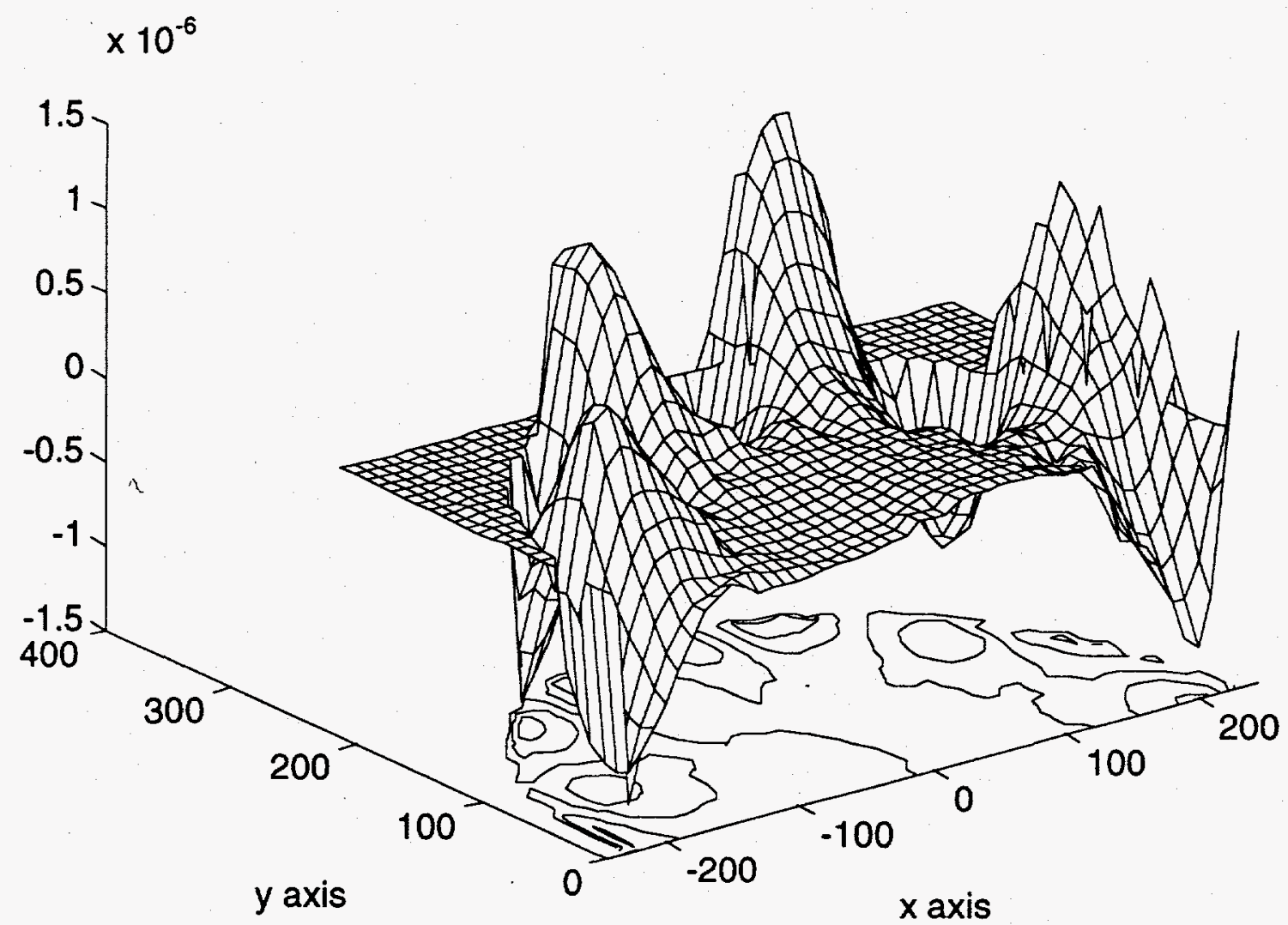

Figure 17: Residual errors for X-gravity and nine-point perimeter support. All axes are in millimeters. One half of the primary mirror is shown.

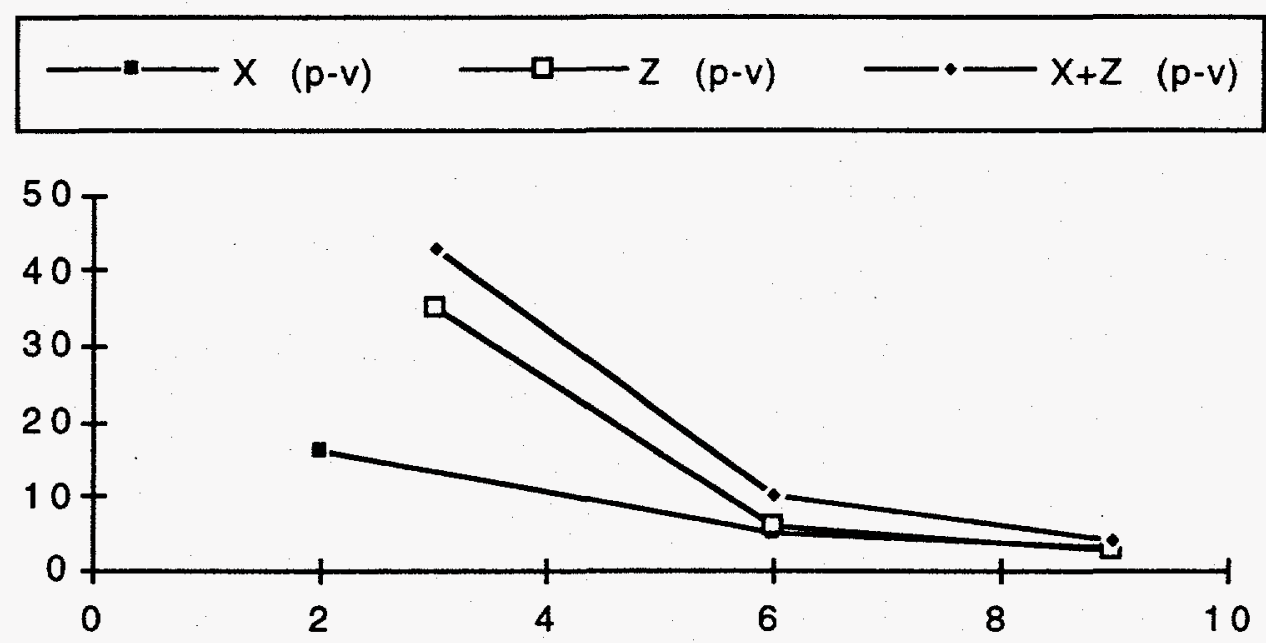

Figure 18: Nonsymmetric error vs. number of supports for $\mathrm{X}, \mathrm{Z}$ and combined gravity load cases.

\section{Sensitivity to Uncertainties}

The sensitivity of the primary mirror to uncertainties present in the support system was not directly calculated; however, it is possible from the previous analyses to infer the errors for force and moment variations. Consider the case where all nine weight-relief forces are 
equal, but their total is slightly different from the weight of the mirror. Since the difference will react against the three-point exact-constraint support system, the results of that analysis can be proportioned by the relative force variation. Expressed as a specification, the relative force variation to cause $1 \mathrm{~nm}$ of additional error is one part in 43 or $2.3 \%$. This translates to about 1 newton of variation per support.

A similar proportioning of the relationship between the $\mathrm{X}$-gravity error and the number of supports can be applied to moments after having made a judgment about the lever arm. Assuming that our hypothesis is correct, then the lever arm would be less than the axial distance between the center of gravity and the midpoint at the perimeter, which is $6.3 \mathrm{~mm}$. The X-gravity error divided by the support force is nearly constant for the three analyses presented. The average of these is $0.078 \mathrm{~nm} /$ newton. Combining this with the lever arm, the sensitivity is greater than $0.012 \mathrm{~nm} / \mathrm{newton} / \mathrm{mm}$. Expressed as a specification, the applied moment to cause $1 \mathrm{~nm}$ of additional error must be safely below 81 newton-mm.

\section{Design of Components}

This section presents the thought process behind the lens design on a more detailed component level than that offered in the Design Overview. The components treated in this section include those of the primary mirror support and adjustment systems, the lens housing, and its support system. Any hindsight thoughts or observations about the prototype designs are provided as possible ways to improve future designs.

The primary mirror support system consists of three main components: a system of flexural links to provide six degrees of constraint; a system of nine pneumatic diaphragm actuators and linkages to provide weight relief; and a welded steel mirror cell to provide structural support for the actuators and flexures. The complete system provides a way to support the mirror with acceptable distortion but without undue care in mounting it to the lens, or for example, during inspection.

The primary mirror adjustment system consists of five main components that support the mirror cell: a system of flexural links to provide three nonadjustable degrees of constraint; three Invar spacing rods to provide three adjustable degrees of constrain; three temporary micrometers to set the axial position of each rod; three sleeve clamps to secure the adjustment of each rod; and three pneumatic actuators to generate strain in each rod for even finer adjustment.

\section{Mirror Flexures}

A flexure provides compliance (or motion) in certain preferred directions while maintaining stiffness (or resistance to motion) in other directions. It works on the principle that a thin beam is very compliant in bending compared to its axial compliance. This ratio (moment to axial compliance) varies inversely as the square of the beam thickness in the direction of bending. The desire to maximize the ratio will invariably require that the width be much larger than the thickness so there is sufficient cross sectional area to carry the load or to provide axial stiffness. Generally, the size constraint on a blade flexure will be either the maximum width or the minimum thickness. The length of the blade may depend on the need for more moment compliance or for less axial compliance, since both are proportional 
to the length. The length to thickness ratio must not be so great as to risk buckling, which for typical materials is approximately 10:1. Equations 6,7 and 8 give the axial, moment and torsional stiffnesses, respectively, for a single blade flexure. Equation 9 gives the condition required for the blade to yield before buckling. The geometric parameters $t, w$ and $a$ represent thickness, width and length, respectively. The material parameters $E, v$ and $\sigma_{y}$ represent elastic modulus, Poisson's ratio and yield strength, respectively.

$$
\begin{gathered}
k_{a}=\frac{E \cdot t \cdot w}{a} \\
k_{m}=\frac{E \cdot t^{3} \cdot w}{12 a} \\
k_{t}=\frac{E \cdot t^{3} \cdot w}{12 a}\left[\frac{1}{2(1+v)}\left(4+2.52 \frac{2 t}{w}\right)+\left(\frac{w}{a}\right)^{2}\right] \\
\left(\frac{t}{a}\right)^{2}>\frac{12}{\pi^{2}} \frac{\sigma_{y}}{E}
\end{gathered}
$$

A single blade flexure provides two rotational degrees of freedom whose compliances are given by the reciprocals of Equations 7 and $8 .{ }^{10}$ When blade flexures are combined in series, their compliances add. Since degrees of freedom are compliances, the combination will have the combined number of independent degrees of freedom. For example, the series combination shown in Figure 19 has only three independent rotational degrees of freedom because the blades' torsional compliances align. Conversely, the blades' constraint directions must align in order for the combination to retain that constraint. The two-blade flexure in Figure 19 provides axial constraint and complete rotational freedom in a compact, monolithic unit. The blades can overlap for even greater compactness but with a small sacrifice to the effective blade width.

A translational degree of freedom can be obtained (assuming small motion) by a series combination of two rotational degrees of freedom having parallel but displaced axes. The basic flexural link used for the mirror flexures is formed by cutting the features of Figure 19 into each end of the link. This places four blades along the same axis to provide one axial constraint, one torsional degree of freedom, and two pairs of rotational and translational degrees of freedom. The translational compliance is related to the rotational compliance by the square of the distance between axes. The ratio of translational to axial compliance varies as the square of the ratio of distance to blade thickness, or by using the buckling relation, as the square of the ratio of distance to blade length. This is why two widely spaced short blades are better than one long blade.

\footnotetext{
${ }^{10}$ The transverse stiffness of a short blade flexure is usually not stiff enough to be a constraint nor compliant enough to be a degree of freedom.
} 


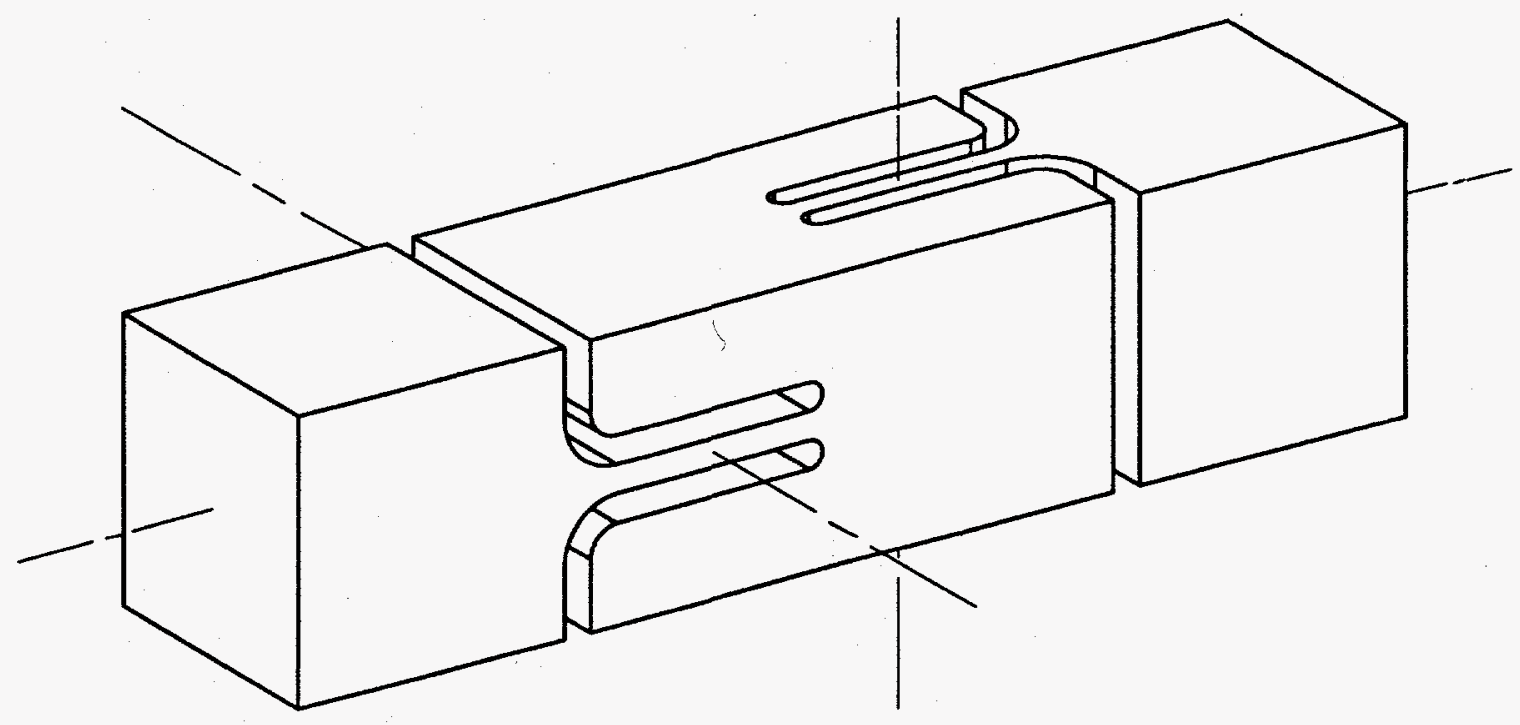

Figure 19: A series combination of blade flexures provides three independent rotational degrees of freedom and one axial constraint. This monolithic flexure can be manufactured using wire EDM.

A parallel combination of flexures will have a combined stiffness equal to the sum of their individual stiffness. Since constraints are stiffnesses, the combination will have the combined number of individual constraints. Figure 20 demonstrates the parallel combination of two flexural links arranged in a vee to constrain the intersection from translating in the $x-y$ plane (the ends are fixed). Three such vee flexures, when connected to the primary mirror as indicated in Figure 2, provide exactly six degrees of constraint.11

Figure 20 was taken from the design program that was developed in Mathcad specifically for this application. The inputs to the program are the geometric parameters shown, the elastic modulus and Poisson ratio, the forces in $x$ and $y$, the displacement in $z$, and the rotations about $x, y$ and $z$. The program calculates a number of outputs that are useful to size the flexure. Among these is the stiffness matrix shown in the figure. ${ }^{12} \mathrm{It}$ shows that the stiffness in the $\mathrm{x}$-direction (70.161) is greater than in the $\mathrm{y}$-direction (41.316). This is desirable because three vee flexures share equally in the mirror's axial stiffness, while the effective number in the plane of the mirror is only 1.5 . An included angle of $110^{\circ}$ would give a $2: 1$ stiffness ratio; however $105^{\circ}$ was chosen to favor slightly the axial direction.

\footnotetext{
${ }^{11}$ It is possible to arrange constraints so they conflict one another. This may be useful to constrain the torsional freedom of an open structure. A misapplication may result in a structure being both over constrained and under constrained.

${ }^{12}$ The columns from left to right are the applied $x-y-z$ translations and $x-y-z$ rotations. The rows from top to bottom are the resulting $x-y-z$ forces and $x-y-z$ moments. You must multiply rotations by $L$ and divide moments by $L$ to arrive at the proper units.
} 


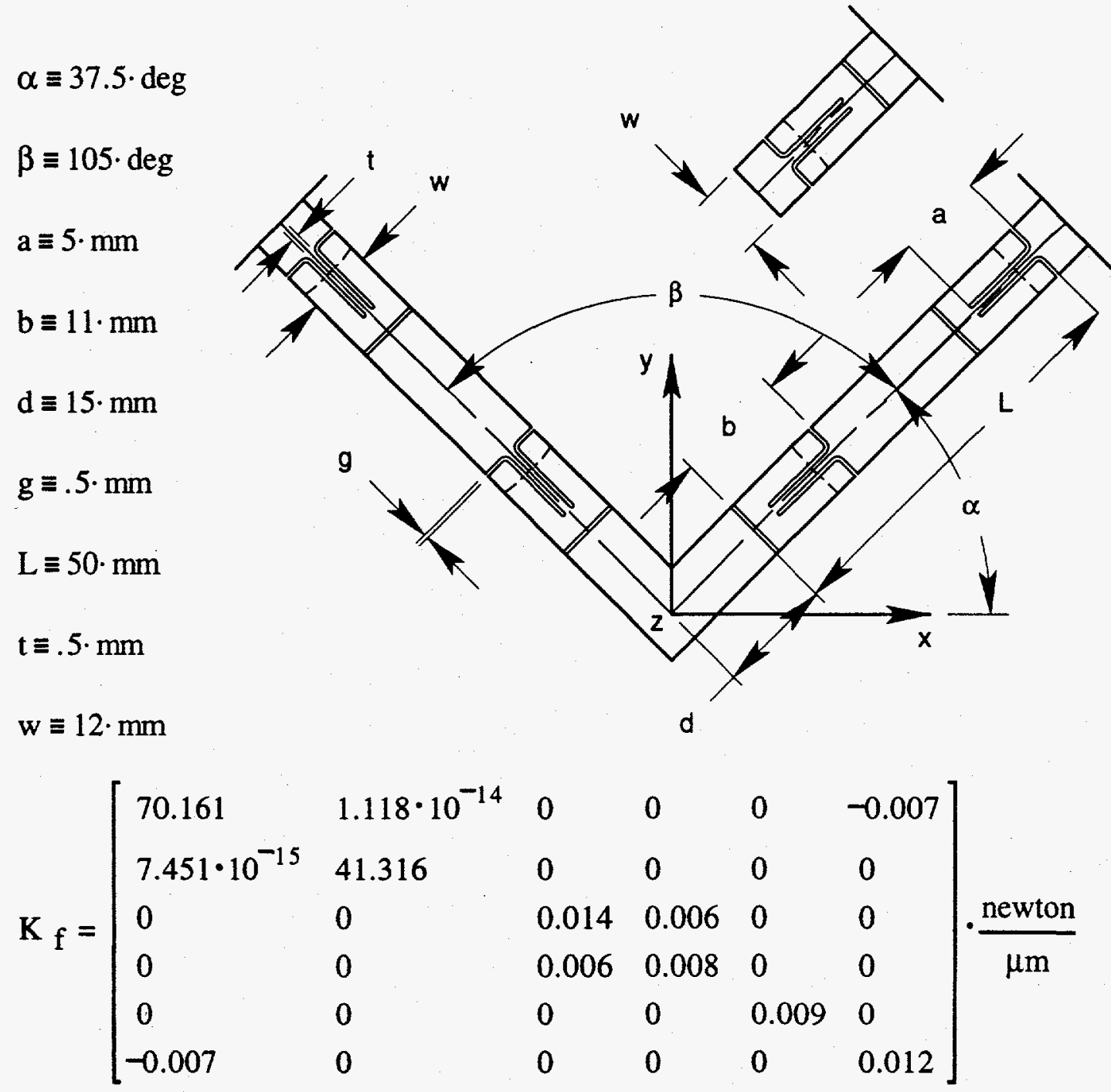

Figure 20: The vee flexure constrains the intersection from translating in the $x-y$ plane. All other directions are relatively free judging from the elements of the stiffness matrix $\mathrm{K}_{\mathbf{f}}$.

Figure 21 shows one vee flexure connected to the primary mirror through a spherical post. The neck and flange of the post were sized using FEA to achieve an allowable stress in the relatively weak Invar 36 and a nearly uniform stress distribution through the epoxy interface to the mirror. The reason to use a spherical joint is to eliminate any forced congruence in the flexure that would generate a moment on the mirror. The joint, however, is not friction-free so careful assembly is necessary. To judge this sensitivity, the limiting coefficient of friction may be calculated from the load carried by the flexure (assume $1 / 3$ of mirror's weight during assembly or 126 newtons), the radius of the sphere $(8 \mathrm{~mm})$ and the allowable moment calculated previously (less than 81 newton-mm). The coefficient of friction must be safely below 0.08 , which is quite low and will require a lubricant or a tapping action to break the friction. In addition, the act of securing the joint must not induce this level of moment back into the flexure. 


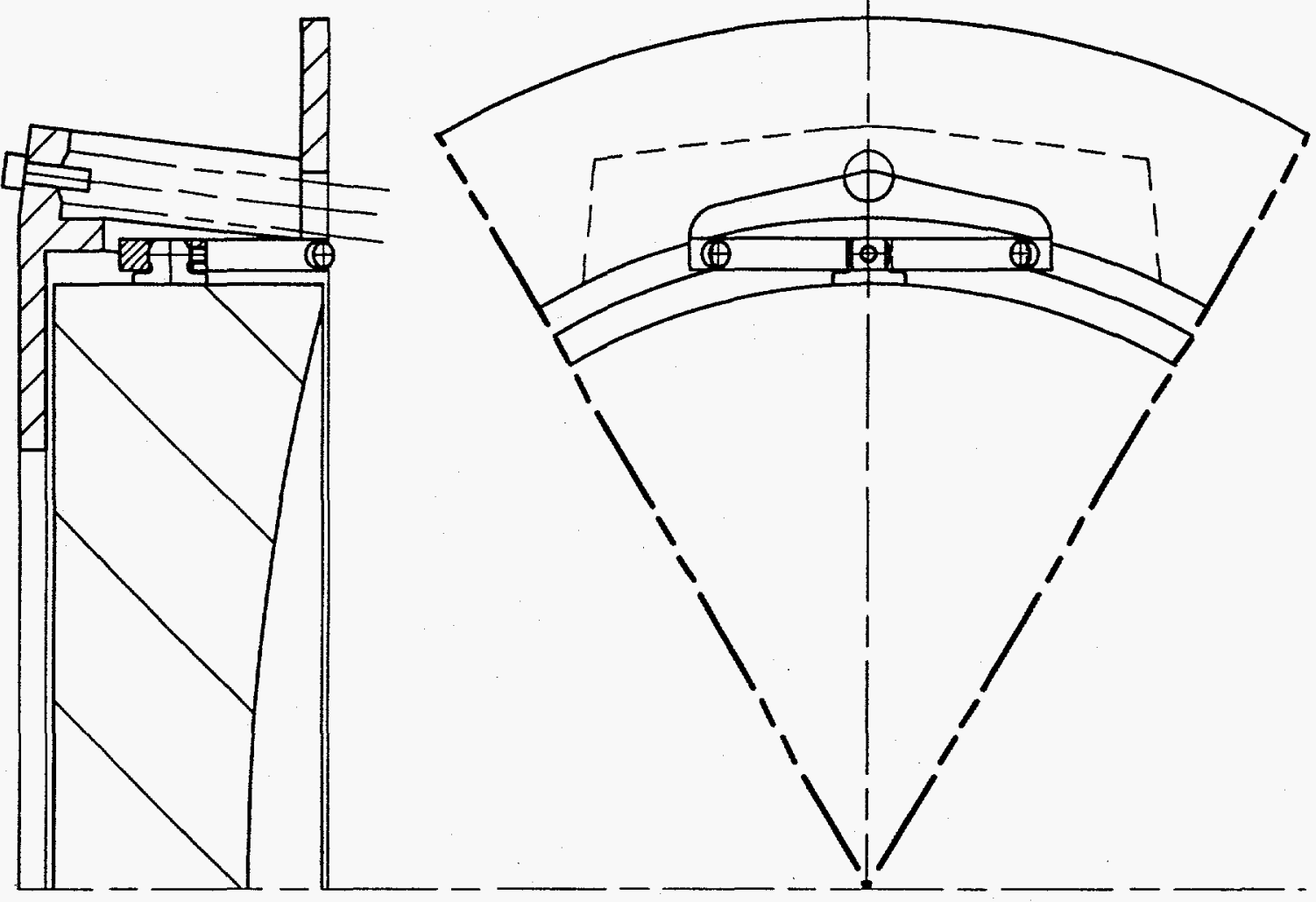

Figure 21: The vee flexure attaches to a post that is bonded to the mirror and its legs attach to the mirror cell. The spacing rod attaches to the mirror cell near the vee flexure for maximum rigidity. These features occur every $120^{\circ}$ around the mirror's perimeter.

The spherical joint design shown in Figure 21 is somewhat different from what would become the prototype. Although difficult to see, a key and set screw when removed from each flexure, allow the mirror to be removed with the flexures still attached to the mirror cell. This is an obvious advantage for final figuring but is not necessary because the flexures attach very simply to the mirror cell. Instead the prototype had a collet clamp to provide better contact with the sphere, which required the central portion of the flexure to fully encircle the post. The disadvantage is significant because the act of securing the collet places greater risk of inducing a moment than does tightening a set screw.

A simpler and better solution would be to use a replicant between each post and flexure, which was an earlier concept. The post would be cylindrical and fit into a clearance hole in the flexure. With all flexures attached to the mirror cell and the mirror supported to insure clearances, epoxy would be injected and allowed to set. The flexures, now being 
permanently attached to the mirror, could be susceptible to damage during final figuring. In addition, the weight of the flexure would alter the figure somewhat.

\section{Weight-Relief Mechanisms}

An ideal weight-relief mechanism would provide a set force in the desired direction with zero stiffness and zero hysteresis in all directions. The design goal was to provide nine equal forces all oriented vertically against gravity as shown in Figure 2. Clearly, those on top would have to pull while those on the bottom would have to push. This was achieved approximately by a single pneumatic pressure regulator plumbed to nine diaphragm actuators and linkages. Figure 22 shows a typical actuator and lever that pulls on the mirror with a link that is not shown. Small buttons are cemented to the mirror using a fixture to guarantee accurate location. A small ball on each end of the link engages a socket on the button and likewise on the lever. Although the actuator may require only a few micrometers of stroke during operation, a stroke of several millimeters is essential for assembly purposes.

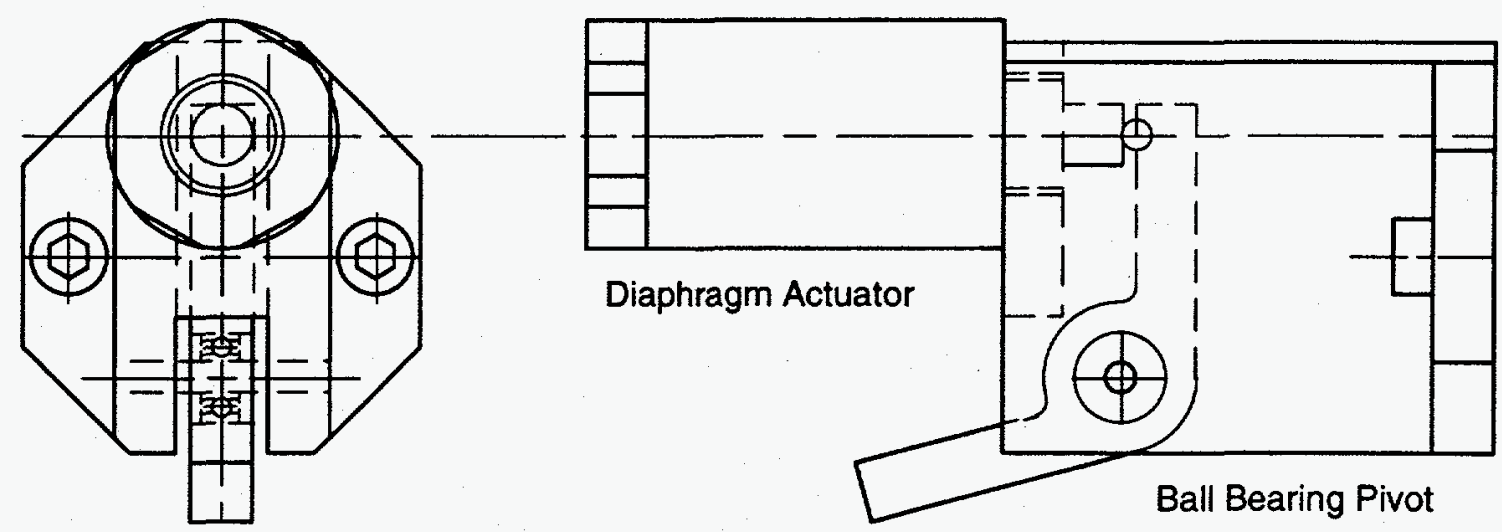

Figure 22: Nine pneumatic actuator and lever assemblies mount to the outer flange of the mirror cell, and through access holes, support the weight of the mirror with simple links.

\section{Mirror Cell}

The mirror cell is a welded steel structure that encircles the mirror and provides support for three vee flexures and nine weight-relief mechanisms. It connects to the rest of the lens through three spacing rods and three tangent flexures. The Z-shaped cross section, shown partially cut away in Figure 21, is relatively easy to fabricate from plate and provides good access for machining and assembly. The mirror cell should have high relative stiffness since it acts in series with other compliances in the fundamental modes of vibration. This was accomplished in the axial direction by locating the spacing rods close to the vee flexures so that the moment arm tending to twist the cell is minimal. In addition, each spacing rod terminates in the corner of a V-shaped brace so that loads are transferred in plane rather than through bending. The cell's compliance is more significant for transverse modes (side-to-side or up-and-down) because the moment arm is larger, which is a squared relationship. Figure 3 shows that the tangent flexures attach to the rear flange of the cell, which was the only area not already occupied by hardware. Dynamic simulations showed 
that the cell's compliance was significant for transverse modes, but it was not a dominating factor as to require a design change.

\section{Micrometer and Clamp Adjustment}

The axial position of each spacing rod relative to the front bulkhead controls the adjustment of the mirror relative to the front optics. As shown in Figure 23, the spacing rod protrudes through a sleeve that threads into the front bulkhead. Both the rod and sleeve are Invar 36. A differential micrometer, connected temporarily to the sleeve, can locate the rod to submicrometer resolution over several millimeters of travel. When the mirror is in proper alignment, the sleeves are clamped to secure the adjustments. The clamp distorts the sleeve into contact with the rod at three places as shown. The clamp and micrometer lie outside the lens housing for easy access, and a threaded cover replaces the micrometer to discourage tampering. We built a demonstration unit to show that sub-micrometer adjustment resolution was possible and that it could withstand loads anticipated during shipping.

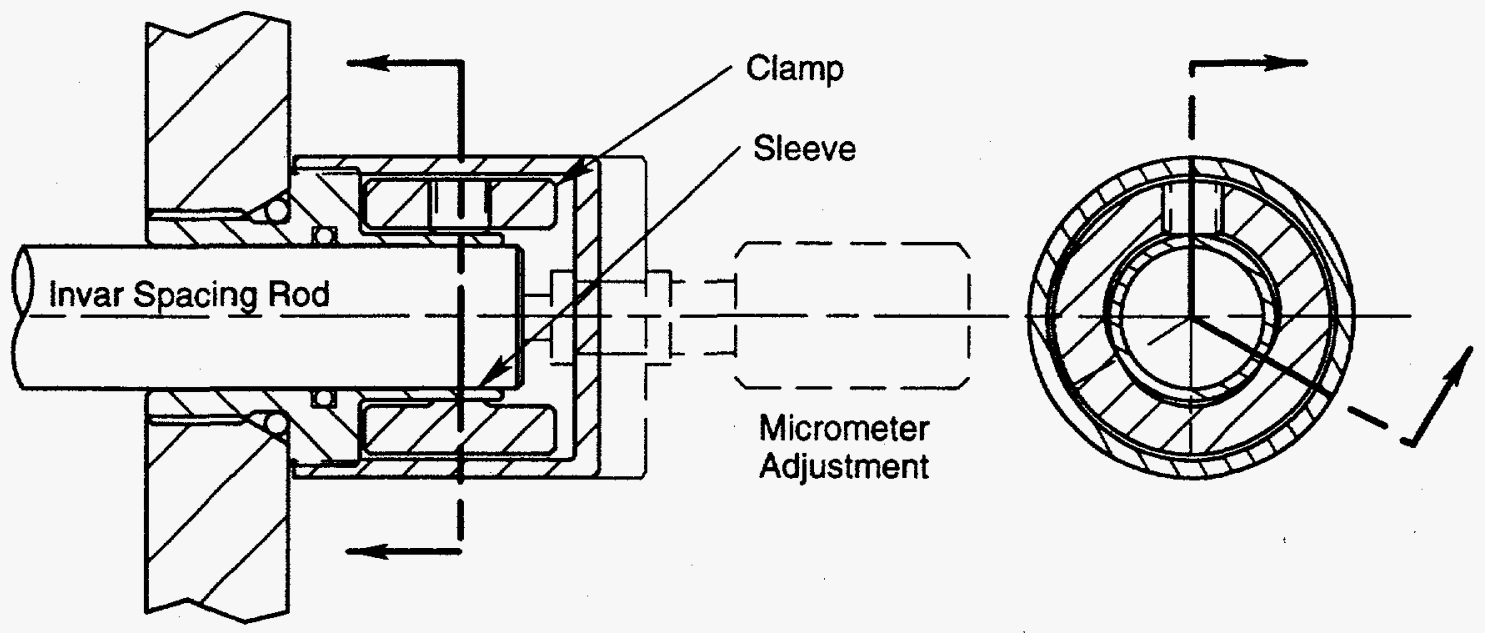

Figure 23: The adjust-and-clamp mechanism provides a resolution of approximately 0.2 micrometer and a frictional force of approximately $1000 \mathrm{lbs}$ for a $3 / 4$ inch rod diameter.

\section{Pneumatic Strain Adjustment}

A fine-tuning adjustment system was provided as a contingency plan in case the micrometer adjustment proved too course. It consists of three bellows actuators and three pneumatic pressure regulators, which act to compress the three spacing rods. Like the weight-relief mechanisms, good force control is essential as relative changes in the pressure cause the same relative changes in displacement. A useful feature of the system is the ability to make adjustments remotely.

\section{Tangent Flexures}

The design of the tangent flexures is a relatively simple extension of the previous work on the vee flexure because it uses the same basic flexural link. The main difference is that three links provide constraint against two translations and one rotation. Figure 24 shows the inputs to the design program and the calculated stiffness matrix. The positions of the flexures are defined to be tangent to a circle of radius $R$ and angled according to the three 
elements of $\alpha$. Two sets of blade parameters are provided to meet the significantly greater motion required in the $\mathrm{z}$ direction for adjustment of the mirror. By adjusting the parameters, it was easy to arrive at a design that met all the requirements, which included matching the lateral stiffness to the combined axial stiffness of the three spacing rods.

$$
\begin{aligned}
& \mathrm{R} \equiv 9.18 \cdot \mathrm{in} \\
& \alpha \equiv\left(\begin{array}{c}
0 \\
120 \\
240
\end{array}\right) \cdot \mathrm{deg} \\
& \mathrm{a} \equiv\left(\begin{array}{c}
.25 \\
.5
\end{array}\right) \cdot \text { in } \\
& \mathrm{b} \equiv 1 \cdot \text { in } \\
& \mathrm{d} \equiv 1 \cdot \text { in } \\
& \mathrm{g} \equiv\left(\begin{array}{c}
.075 \\
.05
\end{array}\right) \cdot \text { in } \\
& \mathrm{L} \equiv 6.125 \cdot \text { in } \\
& \mathrm{t} \equiv\left(\begin{array}{c}
.075 \\
.05
\end{array}\right) \cdot \text { in } \\
& \mathrm{w} \equiv\left(\begin{array}{c}
.5 \\
.75
\end{array}\right) \cdot \text { in }
\end{aligned}
$$

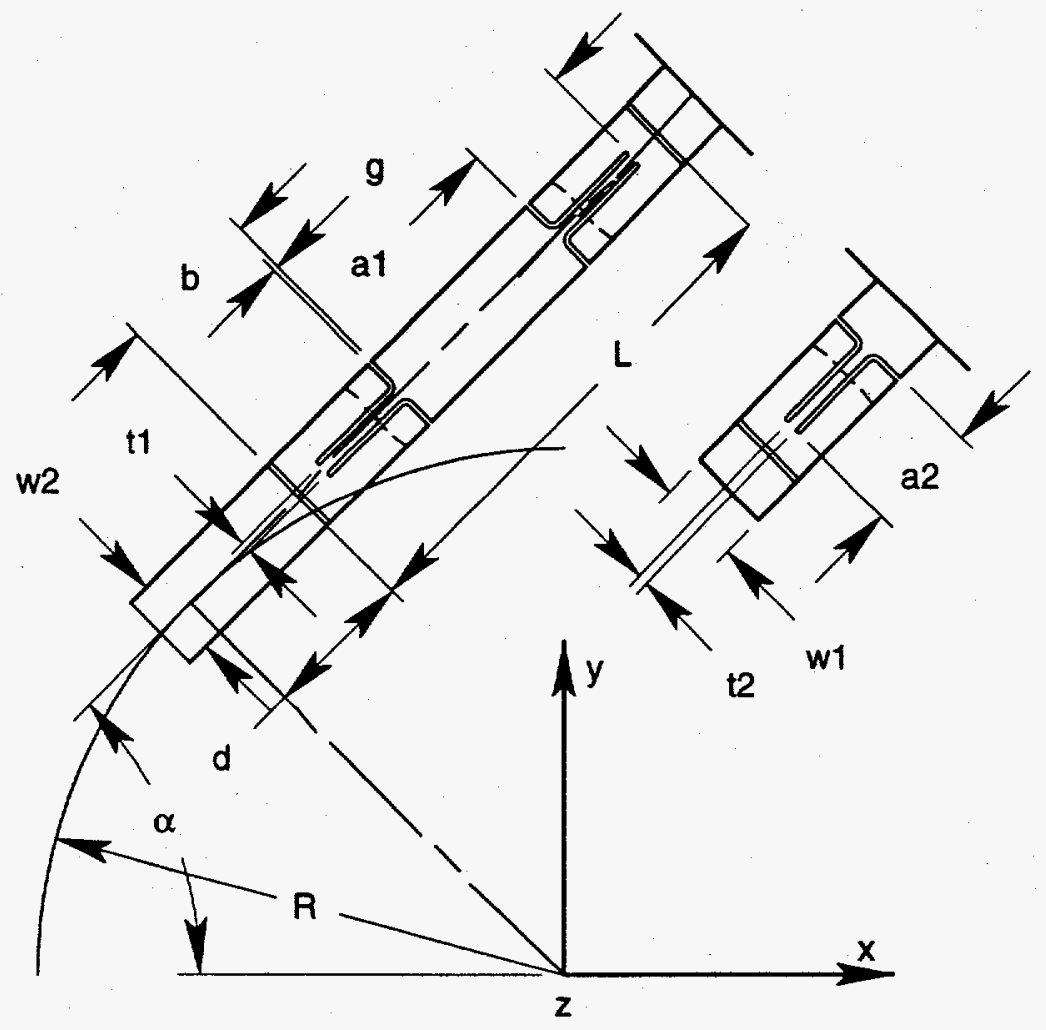

$$
\mathrm{K}_{\mathrm{f}}=\left[\begin{array}{llllll}
182.56 & -4.47 \cdot 10^{-14} & 0 & 0 & 0 & -1.49 \cdot 10^{-14} \\
-4.47 \cdot 10^{-14} & 182.56 & 0 & 0 & 0 & 2.98 \cdot 10^{-14} \\
0 & 0 & 0.016 & 0 & 0 & 0 \\
0 & 0 & 0 & 0.028 & 0 & 0 \\
0 & 0 & 0 & 0 & 0.028 & 0 \\
-2.98 \cdot 10^{-14} & 2.98 \cdot 10^{-14} & 0 & 0 & 0 & 819.983
\end{array}\right] \cdot \frac{\text { newton }}{\mu \mathrm{m}}
$$

Figure 24: The three tangent flexures combine to constrain translation in the $x-y$ plane and rotation about $z$. The principle motions required of the flexures are translations in $\mathrm{z}$ to allow for adjustment of the mirror.

\section{Lens Housing and Support System}

The lens housing, being a structurally closed polyhedral shell, is very rigid to loads applied in the plane of the shells. In cases where loads transfer through bending, the shells require heavy reinforced to maintain rigidity, rather than strength. The front bulkhead, as an example, is subject to bending loads because the spacing rods intersect nearly perpendicular to its surface. It was made substantially thicker than the outer shell and locally reinforced 
with gussets. The support points to the housing also required reinforcement by additional shells and beams.

The desire for low weight and the need for relatively thin shells and local reinforcements made an aluminum casting the first preference. A welded structure was chosen instead to meet the tight schedule and because the design would likely change somewhat when standardized. However, steel was chosen over aluminum because Ultratech was having good results with a similar housing and because there is a factor of two advantage in the thermal coefficient of expansion. Also considered but not investigated was the possibility of a magnesium casting for even lower weight and better damping.

Six adjustable-length rods support the housing and provide adjustment in six degrees of freedom. When designing a linkage system, it is convenient though not essential to arrange pairs of links to lie in common planes, which occurs naturally when there is a plane of symmetry. Any pair (or triple) can be conceptualized as an instant center of rotation, whereby the constrained body acts as if the instant center is a virtual pivot. Instant centers lie at the apparent intersections of lines drawn through the links. Parallel links have an instant center lying at infinity so that motion about that center appears as a translation. Furthermore, the instant center is an abstraction of the viewing plane since the instant center may appear in different locations, perhaps at infinity, for differing views. A three-view drawing is generally sufficient to represent the system, although auxiliary views may facilitate the design process.

Figure 25 shows a schematic representation of the support system used for the prototype lens housing. Each rod is labeled 1 through 6 for identification purposes. Pairs of rods whose extensions truly intersect are indicated by hyphenated numbers corresponding to the rod numbers. Other instant centers that are apparent intersections are not shown to reduce clutter. For example, the intersection between 2 and 4 would appear at a different location in each view. The arrangement of rods was arrived at very simply and without much thought towards the dynamics of the system. The rods were inclined $45^{\circ}$ from the horizontal plane of the stepper base simply for convenience in manufacturing. The rods were grouped in pairs to form three inverted vees with each vee lying in a vertical plane. These planes were arranged to form an equilateral triangle when viewed from above and placed around the lens to provide two vees under the more massive end of the lens. This arrangement provides equal stiffness for any direction in the horizontal plane, with the center of stiffness being at the center of the equilateral triangle and in the plane formed by the instant centers 1-2, 3-5 and 4-6. The vertical stiffness is twice as great (an inclination of $35^{\circ}$ would give equal stiffness), with the center of stiffness being at the center of the triangle formed by the same instant centers. The horizontal and vertical centers of stiffness are indicated in the figures by $\mathrm{H}$ and $\mathrm{V}$, respectively. 

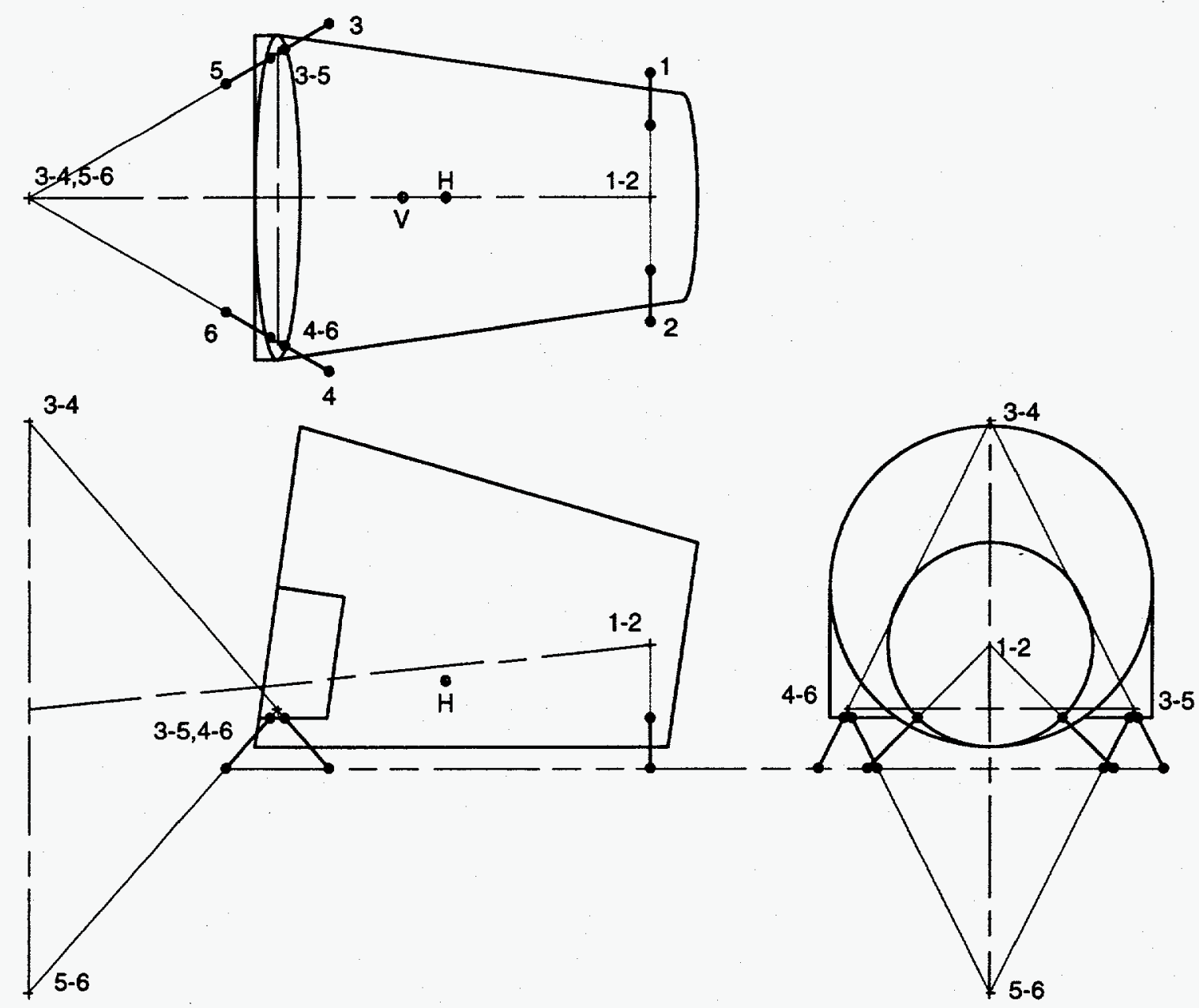

Figure 25: The instant center locations for the prototype lens support (not to scale) are indicated by hyphenated numbers corresponding to the rod numbers. Centerlines indicate axes about which the lens would rotate given the release of 1-2, or in the case of the sloping line, a moment about a horizontal line.

The placement of the horizontal center of stiffness below the mass center causes translational and rotary modes of vibration to couple, which results in lower frequency modes that appear in the simulation to pivot just below the lens. In principle, it is possible to take advantage of the housing stiffness to spread the vees so that their center of stiffness acts near the mass center. This would tend to decouple those modes and would raise the modal frequencies, provided that the additional compliance of the housing is not significant. Figure 26 shows an alternative arrangement where the instant centers appear to be in more favorable positions. We did not, however, evaluate the alternative with a modal analysis. The most apparent change is the single vee placed to the rear (left) of the lens because its instant center is easiest to raise. The two angled vees in front are spread somewhat to raise their instant centers. The net effect is to raise the horizontal center of stiffness as desired, but the vertical stiffness moved forward which may introduce some coupling. 

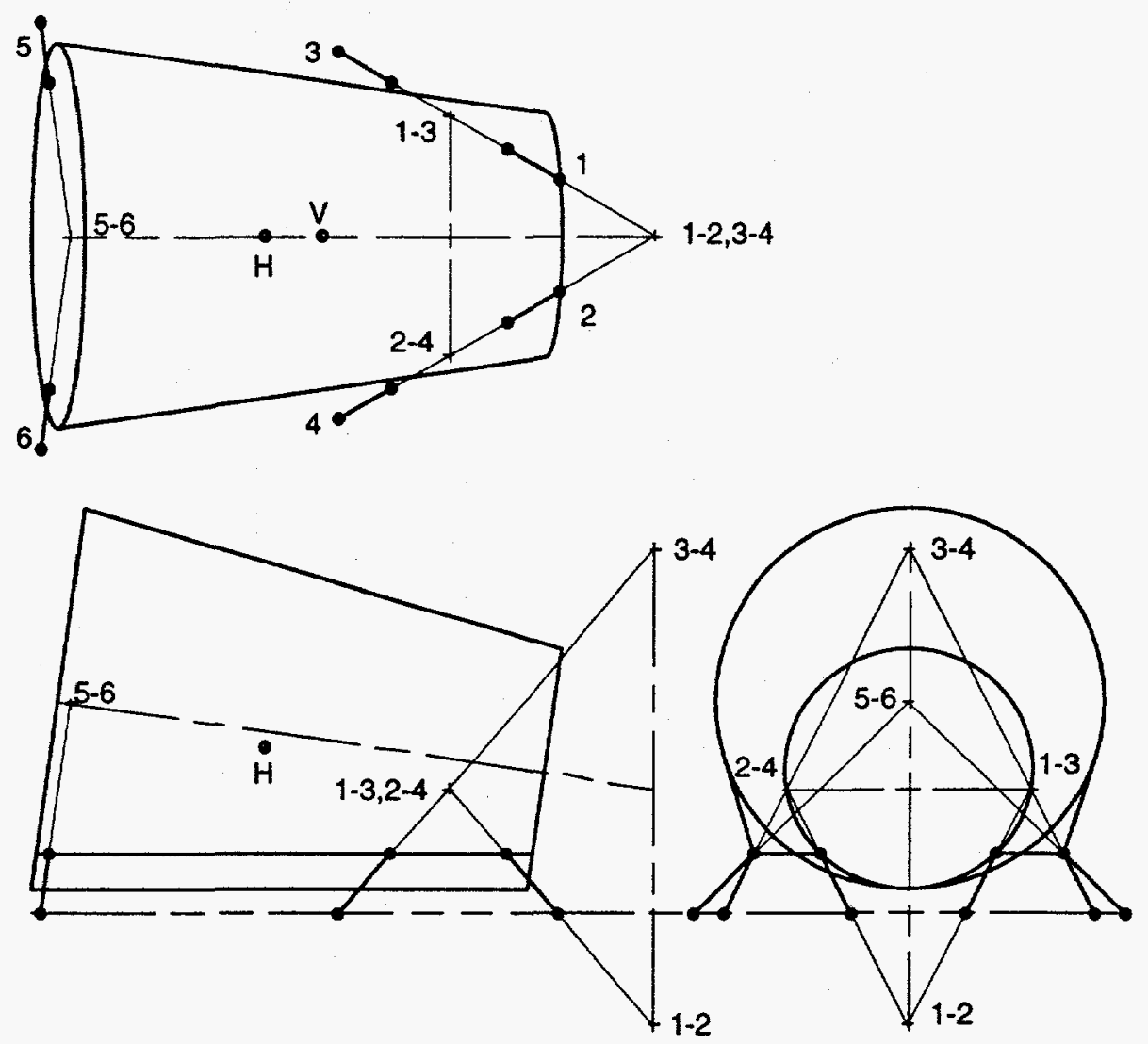

Figure 26: An alternative set of instant centers locations may provide better dynamic performance. The sloping elastic axis nearly coincides with the lens' principal axis, thus lowering the mass moment of inertia.

\section{Dynamic System Model}

A dynamic system model is useful to judge the performance of the new lens system in the presents of vibrations due to stage accelerations and other machine or environmental sources. A modal analysis using the FEA product Applied Structures by Rasna Corp., provided the system model, which includes all major components such as the primary mirror, the cell, the housing, and the all the linkages. Each of the quoted natural frequencies has a convergence percentage or accuracy estimate shown in parentheses, and this value is important to keep in mind when comparing modal frequencies. The goal of the analysis is to demonstrate that the lowest natural mode is above $100 \mathrm{~Hz}$. Figure 27 shows the complete system model.

The analysis was completed in steps so that each subset of the complete system could be understood and optimized, and then integrated into a larger system model. The first step was to model the mirror and vee flexures as if the cell was rigid. The model of the mirror had solid elements with beam elements used to represent the spherical posts. Connected to the posts were beam elements with the appropriate axial stiffness for the flexures and with their ends fixed. The lowest modal frequencies and mode shapes of this analysis are the upper limit for the performance of the complete system, since additional structures add compliance to this subsystem. The first two mode shapes, at $236(5.9 \%)$ and 
$237 \mathrm{~Hz}(5.9 \%)$, were translations of the mirror perpendicular to the optical axis in the vertical and horizontal planes. The third mode is a piston mode of the mirror on the vee flexures at $263 \mathrm{~Hz}(4.4 \%)$. An analytical calculation of the natural frequencies for this configuration, considering only the mirror mass and vee flexure compliance, predicted 265 and $285 \mathrm{~Hz}$ for the same respective modes. The difference is due to the extra compliance of the spherical posts and the mirror itself.

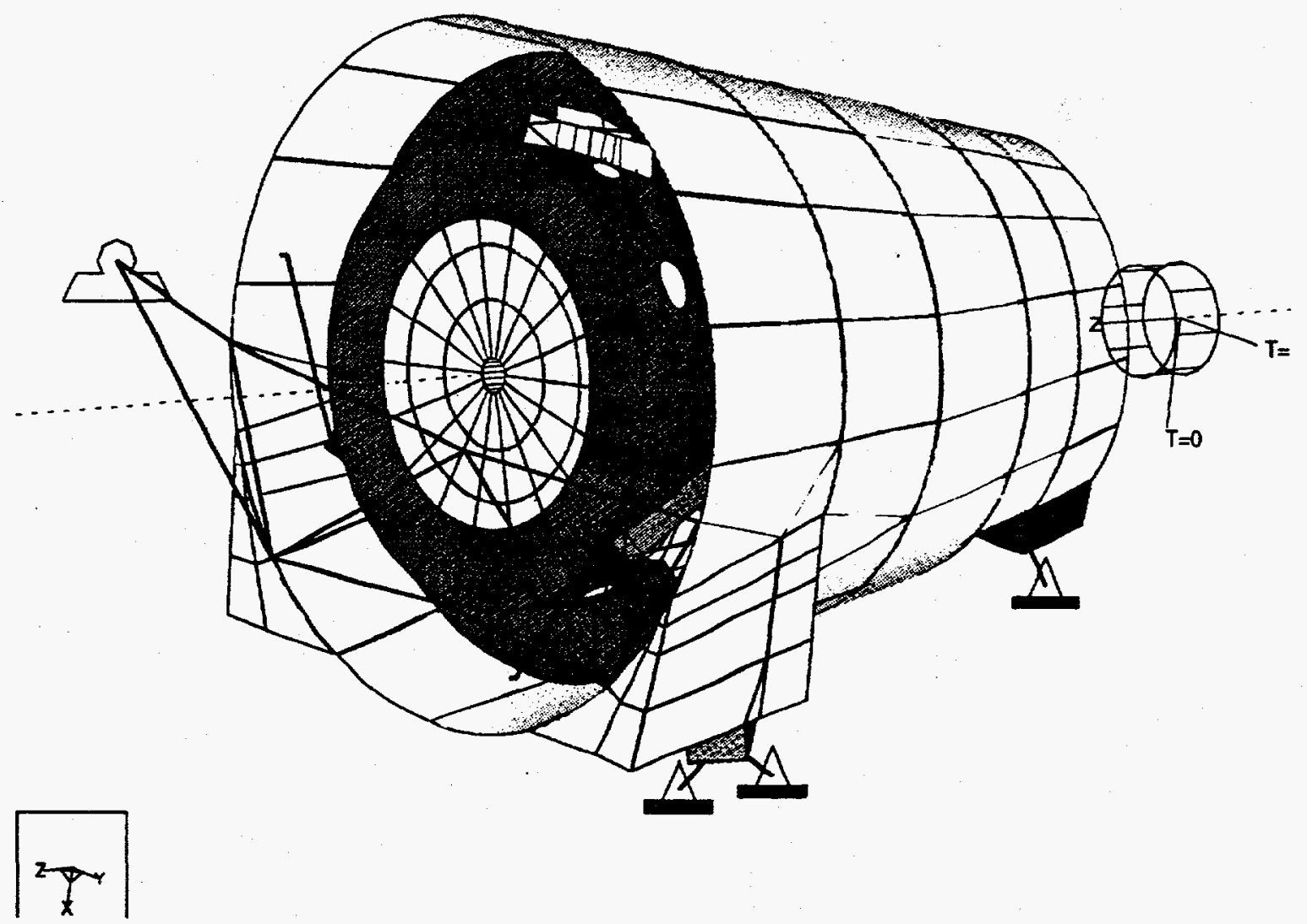

Figure 27: With the rear cover removed, the mirror, mirror cell and tangent flexures are visible inside the housing. To the left is a mass representing the WAS. Three of the six adjustable-length rods and constraints are visible to the lower right.

The second step was to add the cell constrained as if the tangent flexures and spacing rods were axially rigid. The beam elements previously fixed were connected to a shell element model of the cell. The first two mode shapes, now at $185(9.5 \%)$ and $187 \mathrm{~Hz}$ (9\%), are the same translations accompanied by a twisting deformation of the cell. Our hypothesis was that these modes may be improved by connecting the tangent flexure to the cell at locations that alternate with the vee flexures, rather than at the same angular position. However, this had little effect on the model so the tangent flexures remained aligned to the vee flexures in the final design. While the piston mode dropped slightly to $248 \mathrm{~Hz}(3.5 \%)$, the differences in transverse modes indicate that the cell compliance is significant. This problem is addressed in the next model.

The third model had the spacing rods and the tangent flexures added with their free ends fixed. Adding these compliances to the model reduced the frequencies of the first two modes to $137 \mathrm{~Hz}(8 \%)$, but the mode shapes are very similar to the second model, with the 
mirror and cell moving in phase side-to-side and up-and-down and the cell twisting slightly. We made an attempt to reduce the amount of twist occurring in the cell by thickening the cell flanges and by reducing the inside diameter of the hole that opens to the back of the mirror. These modifications had little effect and significant improvement may require closing the section in a circular fashion, thus producing a circular box beam to better resist torsional inputs. The compliances of the spacing rods also brought the mirror piston mode down to $189 \mathrm{~Hz}(3 \%)$. In this mode, the mirror and cell oscillate in phase along the optical axis.

The next model step added the steel housing around the existing model, with the housing fixed in space at its mounting points. This analysis showed little change in the first two mode shapes, where the mirror moves in side-to-side and up-and-down motions. The natural frequencies for these modes dropped only to $130 \mathrm{~Hz}(13 \%)$ and $132(13 \%)$, within the frequency convergence band of the previous $137 \mathrm{~Hz}$ value. The piston mode dropped to $157 \mathrm{~Hz}(5 \%)$. It is similar to that found in the previous model, but lower in frequency due to the compliance of the front bulkhead of the housing where the spacing rods (and transmissive optics) mount.

Next, the six adjustable-length rods that constrain the entire housing were added to the model. This produced significant changes in modal frequencies and introduced some new mode shapes. First, the side-to-side mode of the mirror dropped in frequency to 100 $\mathrm{Hz}(6 \%)$ due to the compliance in the adjustable-length rods allowing the housing to oscillate. In the next mode, the entire housing translates along the optical axis and rotates about a horizontal axis (perpendicular to the optical axis) at $101 \mathrm{~Hz}(7 \%)$. These modes are a function of the stiffness of the adjustable-length rods and the housing rod mounts, which are surprisingly compliant and required some design iteration. At $130 \mathrm{~Hz}(11 \%)$, the mirror and cell show the up-down mode, similar to previous analyses. A complementary mode to the $100 \mathrm{~Hz}$ mode occurs at $169 \mathrm{~Hz}(10 \%)$, with the mirror and cell moving side-to-side together but out of phase with the housing. Lastly, a combination mode at $194 \mathrm{~Hz}(6 \%)$ has the housing pitch (the $101 \mathrm{~Hz}$ mode) out-of-phase with the mirror and cell combination.

We made two attempts to increase these natural frequencies by reducing mass and increasing stiffness. In the first vein, a model with an aluminum housing was analyzed with everything else held constant. This showed no improvement without further stiffening the housing mounts because the reduction in density is offset by a comparable reduction in elastic modulus. With proper reinforcement an aluminum housing should show some improvement while not adding significantly to the cost of fabrication. Larger adjustablelength rods $(0.625$ inch vs. 0.5 inch) were analyzed with the idea to increase the stiffness of the system to ground. Again the modification had little effect on the analysis when perhaps a 25 percent improvement in some modes could be expected due to the increased rod cross-sectional area. This would suggest that the as-modeled housing-mount compliance dominates the rod compliance, thereby mitigating the expected improvement. The physical system may be somewhat stiffer than the model predicts.

Finally, the complete system (assuming a rigid machine base) was modeled by adding the Wafer Alignment System (WAS) and a representative mass for the front transmissive optics. The WAS was modeled as a mass attached at the end of a frame made of 2 inch by 4 inch and 1 inch by 3 inch steel tubing and fixed to the back of the housing, 
as shown in Figure 27. The front transmissive optics were modeled as a mass located with beam elements off the front bulkhead. The effect of these changes was minimal for the first three modes, now at $93 \mathrm{~Hz}(6 \%), 95 \mathrm{~Hz}(6 \%)$, and $128 \mathrm{~Hz}(12 \%)$, and on the last mode at $192 \mathrm{~Hz}(6 \%)$. Mode 4 dropped in frequency from $164 \mathrm{~Hz}$ to $145 \mathrm{~Hz}(10 \%)$ due to the additional rotary inertia of the WAS (about the optical axis) causing the housing to vibrate from side-to-side at the lower frequency. In this analysis, a mode appeared at $161 \mathrm{~Hz}$ $(6 \%)$, which was caused by the WAS swinging in a horizontal plane forcing the center of the back plate to deflect. In the actual hardware, this may not occur because the WAS support is a large 4 inch by 4 inch steel beam that attaches to the housing only at the outer diameter. Table 1 summarizes the six modes from this analysis. A video tape of these modes in animation is available for viewing.

\begin{tabular}{|l|l|l|}
\hline Mode & Freq $(\mathrm{Hz})$ & Description \\
\hline 1 & 93 & Mirror/cell/housing side-to-side motion \\
\hline 2 & 95 & Housing pitches on adjustable-length rods \\
\hline 3 & 128 & Mirror/cell up-down motion \\
\hline 4 & 146 & Mirror/cell side-to-side, out-of-phase w/ housing side-to-side \\
\hline 5 & 161 & Mirror/cell piston, caused by WAS attachment to back plate \\
\hline 6 & 192 & Mirror/cell piston out-of-phase with slight housing pitch \\
\hline
\end{tabular}

Table 1: System model modes and frequencies.

The goal for this phase of the project was to demonstrate a lowest system mode of $100 \mathrm{~Hz}$. While the final model analysis has a lowest modal frequency of $93 \mathrm{~Hz}$, testing of the prototype hardware would demonstrate the true performance of the system. If required, several modifications may be considered to raise this value. An aluminum housing designed to allow for the lower modulus of elasticity of the material so that the existing stiffness could be maintained would result in a mass savings of approximately 50 percent on the housing, resulting in perhaps a 10 percent increase in the natural frequencies of some modes. Several of the modes almost appear to be rigid body motions of the housing, leading to the assumption that increased adjustable-length rod stiffness is desired. In the finite element model, increasing rod stiffness alone did not increase the modal frequencies, leading to the assumption that some compliance must exist in the rod mounts; this may only be true in the finite element model and before modifications to hardware are made, the mount compliance should be compared to the adjustable-length rod compliance. 